\title{
2-1/2-D Electromagnetic Modeling of Nodular Defects in High-power Multilayer Optical Coatings
}

N. E. Molau

H. R. Brand, M. R. Kozlowski, C. C. Shang

\section{RECEIVED \\ SEP 251996 \\ OSTI}

July 1, 1996

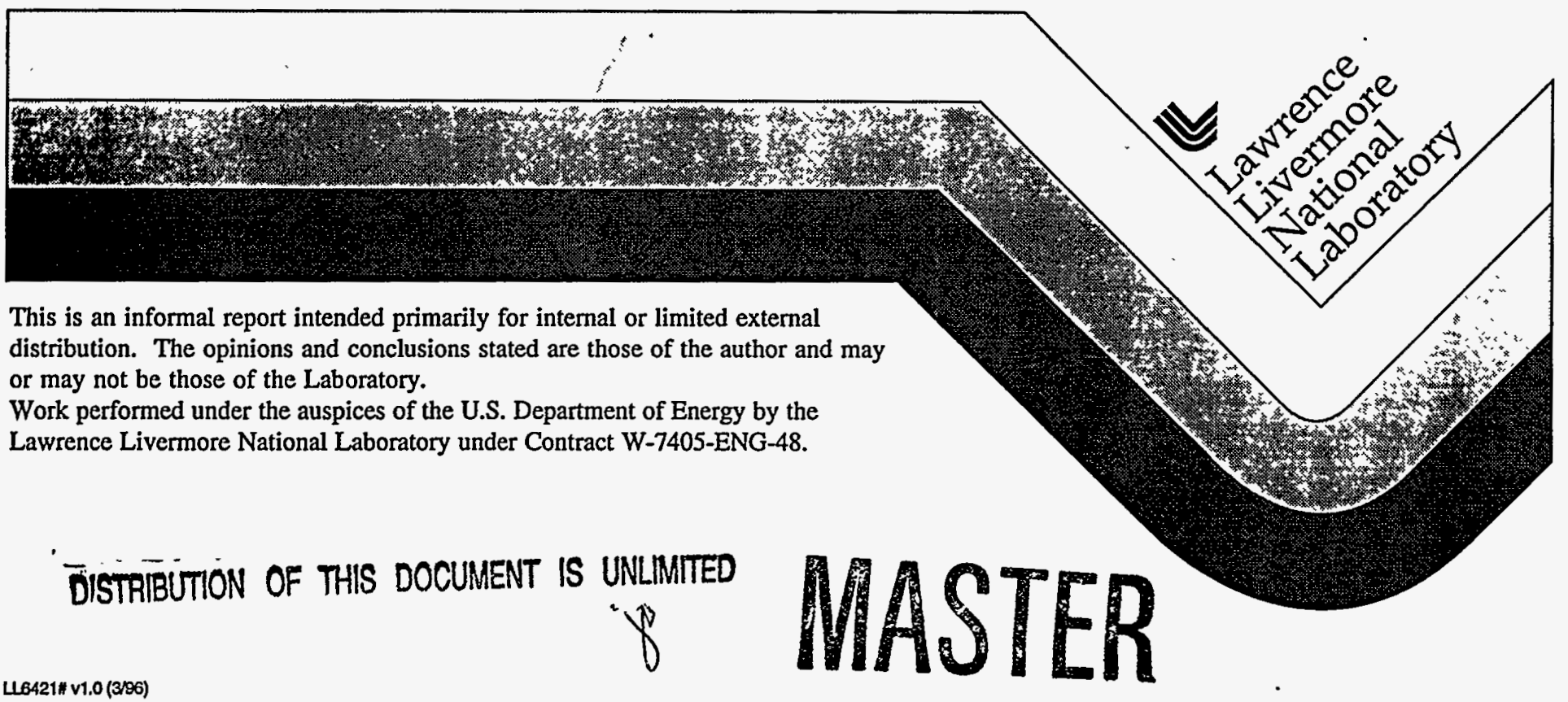




\section{DISCLAIMER}

This document was prepared as an account of work sponsored by an agency of the United States Government. Neither the United States Government nor the University of California nor any of their employees, makes any warranty, express or implied, or assumes any legal liability or responsibility for the accuracy, completeness, or usefulness of any information, apparatus, product, or process disclosed, or represents that its use would not infringe privately owned rights. Reference herein to any specific commercial product, process, or service by trade name, trademark, manufacturer, or otherwise, does not necessarily constitute or imply its endorsement, recommendation, or favoring by the United States Government or the University of California. The views and opinions of authors expressed herein do not necessarily state or reflect those of the United States Government or the University of California, and shall not be used for advertising or product endorsement purposes.

This report has been reproduced directly from the best available copy.

Available to DOE and DOE contractors from the

Office of Scientific and Technical Information

P.O. Box 62, Oak Ridge, TN 37831

Prices available from (615) 576-8401, FTS 626-8401

Available to the public from the

National Technical Information Service

U.S. Department of Commerce

5285 Port Royal Rd.

Springfield, VA 22161

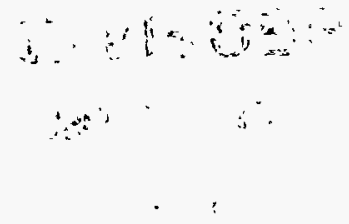




\section{DISCLAIMER}

Portions of this document may be illegible in electronic image products. Images are produced from the best available original document. 
Lawrence Livermore

\title{
2-1/2-D Electromagnetic Modeling of Nodular Defects in High-Power Multilayer Optical Coatings
}

\author{
Project Report
}

N. E. Molau

H. R. Brand, M. R. Kozlowski, C. C. Shang

July.1996 


\title{
2-1/2-D Electromagnetic Modeling of Nodular Defects in High-Power Multilayer Optical Coatings
}

\author{
by N. E. Molau, H. R. Brand, M. R. Kozlowski, and C. C. Shang
}

\section{Introduction}

Advances in the design and production of high damage threshold optical coatings for use in mirrors and polarizers have been driven by the design requirements of high-power laser systems such as the proposed 1.8-MJ National Ignition Facility (NIF) and the prototype 12-kJ Beamlet laser system. The present design of the NIF will include 192 polarizers and more than 1100 mirrors. Currently, the material system of choice'for high-power multilayer optical coatings with high damage threshold applications near $1.06 \mu \mathrm{m}$ are e-beam deposited $\mathrm{HfO}_{2} / \mathrm{SiO}_{2}$ coatings. However, the optical performance and laser damage thresholds of these coatings are limited by micron-scale defects and insufficient control over layer thickness.

In multilayer dielectric stack mirrors, the predominant surface defects are micron-scale domes associated with the classic nodule defect. ${ }^{1,2}$ These defects are initiated at seed particles that are either present on the substrate or that are deposited during the film deposition process. The seed particles can arise from several sources: contamination within the vacuum system, substrate contamination, or particles ejected from the coating source material itself. Nodular defects, similar to those shown in the micrograph in Fig. 1, are produced during film deposition as a result of shadowing effects at seed particles during the growth process. These defects give rise to local electric field enhancements which lead to "hot spots" in the coatings since the heat generation is proportional to the square of the electric field, and these rapidly expanding regions create tensile stresses which may result in mechanical failure of the coatings.

For the past several years, there has been an ongoing effort. at LLNL to use computer modeling as a tool to study and better understand the interaction mechanisms between the laser light and the nodular defects in multilayer dielectric high

\footnotetext{
${ }^{1}$ L. F. Johnson, E. J. Ashley, T. M. Donovan, J. B. Franck, R. W. Woolever, R. Z. Dalbey, “Scanning Electron Microscopy Studies of Laser Damage Initiating Defects in $\mathrm{ZnSe} / \mathrm{ThF}_{4}$ and $\mathrm{SiH} / \mathrm{SiO}_{2} \mathrm{Multilayer}$ Coatings," Laser Damage in Optical Materials: 1984, NBS Special Pub. 727, 1984, p. 356.

${ }^{2}$ M. C. Staggs, M. Balooch, M. R. Kozlowski, W. J. Siekhaus, "In Situ Atomic Force Microscopy of LaserConditioned and Laser-Damaged $\mathrm{HfO}_{2} / \mathrm{SiO}_{2}$ Dielectric Mirror Coatings," Laser-Induced Damage in Optical Materials: 1991, SPIE Vol. 1624, 1992, p. 375.
} 


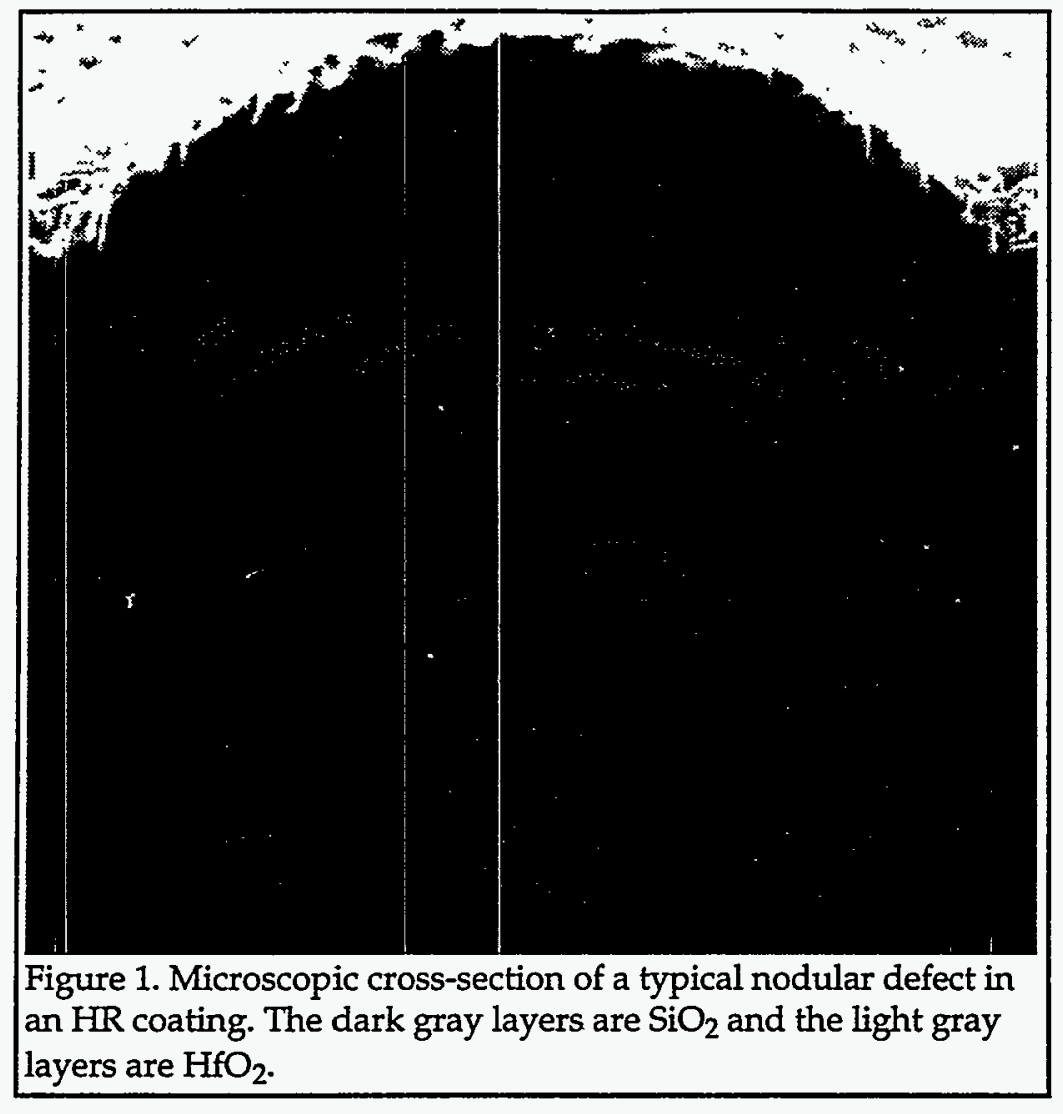

reflectance (HR) coatings. The first 2-1/2-D finite-element, time-domain (FDTD) electromagnetic (EM) modeling of rotationallysymmetric nodular defects in multilayer dielectric coatings at LLNL were performed by DeFord $^{3,4}$ using AMOS, ${ }^{5,6,7}$ a 21/2-D FDTD EM code developed at LLNL, and assuming normal-incidence illumination. They studied the effects of nodule size and depth on the electric field enhancement along the nodule axis of symmetry. Subsequently, Molau ${ }^{8}$ verified some of DeFord's 2-1/2-D AMOS EM modeling results as well as performed a 2-1/2-D AMOS EM analysis of a dielectric stack mirror at a $45^{\circ}$ angle-of-incidence. Upon performing several additional oblique-incidence simulations using rotationallysymmetric test geometries with known analytic solutions, it was demonstrated that while the 2-1/2-D FDTD AMOS EM code could be used to perform oblique-incidence simulations for simple geometries such as the dielectric stack mirror, the code was not, in general, well-suited to performing oblique-incidence simulations of more complex rotationally-symmetric geometries. Shang performed the preliminary FDTD EM

\footnotetext{
${ }^{3}$ J. F. DeFord, M. R. Kozlowski, "Modeling of Electric-Field Enhancement at Nodular Defects in Dielectric Mirror Coatings," Laser-Induced Damage in Optical Materials: 1992, SPIE Vol. 1848 (1993), SPIE Proc. Vol. 1848, 455-473 (1993).

${ }^{4}$ M. R. Kozlowski, J. F. DeFord, M. C. Staggs, "Laser-Damage Susceptibility of Nodular Defects in Dielectric Mirror Coatings: AFM Measurements and Electric-Field Modeling," Proceedings of Second International Conference on Laser Ablation, Knoxville, TN, April 19-22, 1993.

${ }^{5}$ J. F. DeFord, G. D. Craig, R. McLeod, "The AMOS (Azimuthal Mode Simulator) Code," Proceedings of the 1989 Particle Accelerator Conference, Chicago, IL, Mar. 20-23, 1989, P. 1181.

${ }^{6} \mathrm{~J}$. F. DeFord, G. D. Craig, R. McLeod (1990), "The AMOS Wakefield Code," Proc. Conf. on Computer Codes and the Linear Accelerator Community (Los Alamos, NM, January 22-25), 265.

${ }^{7}$ C. C. Shang, J. F. DeFord (1990), "Modified-Yee Field Solutions in the AMOS Wakefield Code," Proc. 1990 Linear Accelerator Conf. (Albuquerque, NM, Sept.).

${ }^{8}$ N. E. Molau, "Summary of the Mirror Defect Modeling Project," LLNL Internal Report, 1994.
} 
simulations on rotationally-symmetric nodules that studied the electric field enhancement throughout an entire 2-D r-z cross-section of the nodular defect and surrounding multilayer dielectric stack rather than just along the nodule axis of symmetry. ${ }^{9}$ Shang used a diagnostic within AMOS that essentially takes a 2-D temporal "snapshot" in time of the electric field within the 2-D r-z cross-section being studied. One limitation of this diagnostic is that it does not give the "hot spots" in the steadystate electric field pattern everywhere throughout the 2-D region, but rather takes a temporal "snapshot" of the steady-state electric field, which may give the peak fields in some locations but not in others, due to relative phase differences between the various "hot spots". in the electric field profiles. Shang examined several of these 2-D temporal "snapshots" over one steady-state cycle to determine the approximate locations of "hot spots" in the electric-field profiles, a time-intensive process. This electric field data was then used to provide the heat generation terms $\left(\sigma \mathrm{E}^{2}\right.$, with $\sigma$, the electrical conductivity and $\mathrm{E}$, the total steady-state electric field amplitude) for the 2-D thermal-mechanical simulations performed by Sawicki ${ }^{10}$ as part of the 2-D coupled electro-mechanical simulation effort for nodular defects in planar multilayer dielectric stack mirrors.

In this report, we will discuss the results of our 2-1/2-D FDTD EM modeling effort for rotationally-symmetric nodular defects in multilayer dielectric HR coatings. We have added a new diagnostic to the 2-1/2-D FDTD EM code, AMOS, that enables us to calculate the peak steady-state electric fields throughout a 2-D planar region containing a 2-D r-z cross-section of the axisymmetric nodular defect and surrounding multilayer dielectric stack. We have also generated a series of design curves to identify the range of loss tangents for $\mathrm{SiO}_{2}$ and $\mathrm{HfO}_{2}$ consistent with the experimentally determined power loss of the HR coatings. In addition, we have developed several methods to provide coupling between the EM results and the thermal-mechanical simulation effort. We now have the capability of coupling the 2-1/2-D EM results into 2$\mathrm{D}$ thermal-mechanical codes such as the commercially available COSMOS/ $\mathrm{M}^{11}$ code or LLNL's 2-D NIKE2D/TOPAZ2D code suite, as. well as into LLNL's 3-D thermalmechanical code suite, NITO3D (comprised of the loosely-coupled NIKE3D and TOPAZ3D codes). We have simulated a matrix of 23 defect geometries to study the effects of seed size, depth and material composition on the electric field enhancement

\footnotetext{
${ }^{9}$ Shang et al., "Coupled-Code Simulation for High-Power Components and Microwave Sources," LLNL Computational Electronics and Electromagnetics Thrust Area Report, 1994.

${ }^{10}$ R. H. Sawicki, C. C. Shang, T. L. Swatloski, "Failure Characterization of Nodular Defects in Multi-layer Dielectric Coatings," Boulder Damage Symposium, Boulder, CO, Oct. 27-29,1994.

${ }^{11}$ COSMOS/M Finite Element Analysis computer code, version 1.70, 1993, Structural Research and Analysis Corporation, Santa Monica, CA.
} 
throughout the 2-D region containing an $x-z$ cross-section of the rotationally-symmetric nodular defect and surrounding multilayer dielectric stack. We will discuss the results of the 2-1/2-D FDTD EM simulations in this report.

\section{Modeling Technique}

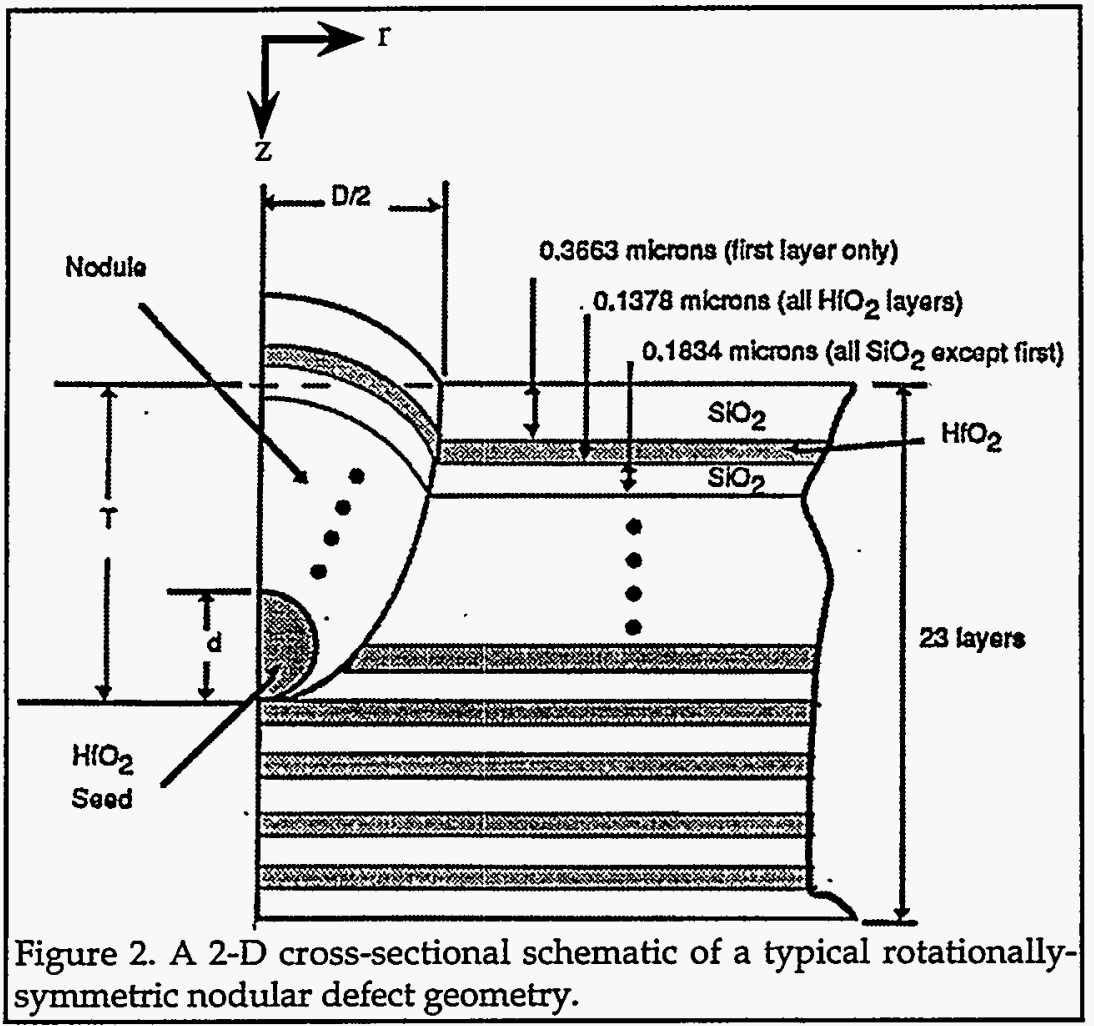

Multilayer dielectric stack HR coatings contain imperfections such as nodular defects that are produced during the film deposition process. We have performed a 2-1/2-D FDTD EM computer analysis of various rotationallysymmetric nodular defect geometries similar to the geometry shown schematically in Fig. 2. Fig. 2 shows a 2-D cross section in the $\mathrm{r}-\mathrm{z}$ plane of a typical 23layer $\mathrm{HfO}_{2} / \mathrm{SiO}_{2} \cdot \mathrm{HR}$ coating containing a $\mathrm{HfO}_{2}$ seed with diameter, $d$, and embedded with some depth, $T$, into the perfect stack, with additional $\mathrm{HfO}_{2} / \mathrm{SiO}_{2}$ layers deposited above the seed, thereby forming a rotationally-symmetric nodular defect of diameter, $\mathrm{D}$, with parabolic sides defined by the relation, $\mathrm{D}=$ $\left(8^{*} \mathrm{~T}^{*} \mathrm{~d}\right)^{1 / 2}$. All of the $\mathrm{HfO}_{2}$ and $\mathrm{SiO}_{2}$ layers are quarter-wave layers (for an optical wavelength of $1.06 \mu \mathrm{m}$ ) with the exception of the half-wave $\mathrm{SiO}_{2}$ overcoat at the air/HR coating interface. The seed material was assumed to be $\mathrm{HfO}_{2}$ in all of the 23 geometries analyzed except one, for which the seed material was changed to $\mathrm{SiO}_{2}$ in order to observe the effect of seed composition on the electric field enhancement. The electrical material properties (i.e., the relative permittivity, $\varepsilon_{\mathbf{r}}$, and the electrical conductivity, $\sigma$ ) used in the computer simulations are shown in Table 1 . The choice of electrical conductivities was such that the loss tangent of $\mathrm{HfO}_{2}$ was twice that of $\mathrm{SiO}_{2}$ for an average power loss of $\sim 150 \mathrm{ppm}$; where the loss tangent is defined as $\tan \delta=\sigma / \omega \varepsilon$, with $\omega$, the radial frequency ( $\omega=2 \pi f$ with $f$, the optical frequency in $\mathrm{Hz}$ ) and $\varepsilon=\varepsilon_{\mathrm{r}}^{*} \varepsilon_{\mathrm{O}}$ with 
$\varepsilon_{0}=8.85 \times 10^{-12} \mathrm{~F} / \mathrm{m}$, the permittivity of free space and $\varepsilon_{\mathfrak{r}}$, the relative permittivity. This choice of loss tangents and electrical conductivities will be discussed in more detail in the next section. The 2-1/2-D FDTD EM simulations assumed normal-incidence illumination with a single-frequency, sinusoidally varying, linearly-polarized, plane wave with an average input power density of $1 \mathrm{GW} / \mathrm{cm}^{2}$.

\begin{tabular}{|l||c|c|}
\hline \multicolumn{1}{|c|}{ Property } & $\mathrm{SiO}_{2}$ & $\mathrm{HfO}_{2}$ \\
\hline \hline Relative Permittivity & 2.1 & 3.73 \\
\hline Electrical Conductivity $(\mathrm{S} / \mathrm{m})$ & 0.3366 & 1.1956 \\
\hline Loss Tangent & $10.2 \times 10^{-6}$ & $20.4 \times 10^{-6}$ \\
\hline
\end{tabular}

Table 1. Electrical material properties used in the EM analyses.

The 2-1/2-D FDTD EM code, AMOS, was used to study the electric field enhancements in high-power optical coatings containing nodular defects. Because AMOS is a 2-1/2-D code, we were limited to analyzing defect geometries that were rotationally-symmetric and to using normal-incidence illumination. The AMOS code solves the Maxwell equations in cylindrical coordinates in the time domain, using the appropriate boundary conditions ${ }^{12}$ and material models. ${ }^{13}$ The AMOS code decomposes the EM fields into a sum of modes, each assuming a harmonic $\left(\mathrm{e}^{\mathrm{i} \phi}\right) \phi$-variation to reduce the 3-D problem to a 2-D one, and then solves for the mode amplitudes of the fields in the 2-D r-z plane. (This assumption of an implicit $\phi$-dependence is the reason that AMOS is considered a 2-1/2-D code.) Since a rotationally-symmetric object cannot induce azimuthal $(\phi)$ multipole coupling, the source fields and the scattered fields will have the same azimuthal dependence. Therefore, if we assume an incident plane wave propagating in the $+z$ direction and linearly polarized in the $x$-direction, represented in cylindrical coordinates as

$$
\begin{aligned}
& E_{r}^{(i n c)}=E_{o} \cos (\phi) \cos (\varpi t-\beta z), \\
& E_{\phi}^{(i n c)}=E_{o} \sin (\phi) \cos (\varpi t-\beta z), \\
& H_{r}^{(i n c)}=\frac{E_{o}}{\eta_{o}} \sin (\phi) \sin (\varpi t-\beta z), \\
& H_{\phi}^{(i n c)}=-\frac{E_{o}}{\eta_{o}} \cos (\phi) \sin (\varpi t-\beta z),
\end{aligned}
$$

\footnotetext{
${ }^{12}$ Mur, G. (1981), IEEE Trans. Electromagn. Compat., EMC-23, 1073.

${ }^{13}$ Luebbers, R. et al. (1990), IEEE Trans. Antennas Propag., AP-32, 222.
} 
with $\omega$, the radial frequency; $\beta=2 \pi / \lambda_{\mathrm{o}}$, the propagation constant; and $\eta_{0}=377 \Omega$, the intrinsic impedance of free space; then the $\phi$ derivatives in the Maxwell curl equations can be done explicitly, resulting in the following equations (in cylindrical coordinates) which will be satisfied by the scattered fields

$$
\begin{aligned}
& \frac{1}{r} H_{z}-\frac{\partial H_{\phi}}{\partial z}=\varepsilon \frac{\partial E_{r}}{\partial t}, \\
& \frac{\partial H_{r}}{\partial z}-\frac{\partial H_{z}}{\partial r}=\varepsilon \frac{\partial E_{\phi}}{\partial t}, \\
& \frac{1}{r} \frac{\partial}{\partial r}\left(r H_{\phi}\right)-\frac{1}{r} H_{r}=\varepsilon \frac{\partial E_{z}}{\partial t}, \\
& -\frac{1}{r} E_{z}-\frac{\partial E_{\phi}}{\partial z}=-\mu \frac{\partial H_{r}}{\partial t}, \\
& \frac{\partial E_{r}}{\partial z}-\frac{\partial E_{z}}{\partial r}=-\mu \frac{\partial H_{\phi}}{\partial t}, \\
& \frac{1}{r} \frac{\partial}{\partial r}\left(r E_{\phi}\right)+\frac{1}{r} E_{r}=-\mu \frac{\partial H_{z}}{\partial t},
\end{aligned}
$$

with $\varepsilon$, the permittivity and $\mu$, the permeability; and where $\mathrm{E}_{\mathrm{r}}, \mathrm{E}_{z^{\prime}}$, and $\mathrm{H}_{\phi}$ vary as $\cos \phi$,

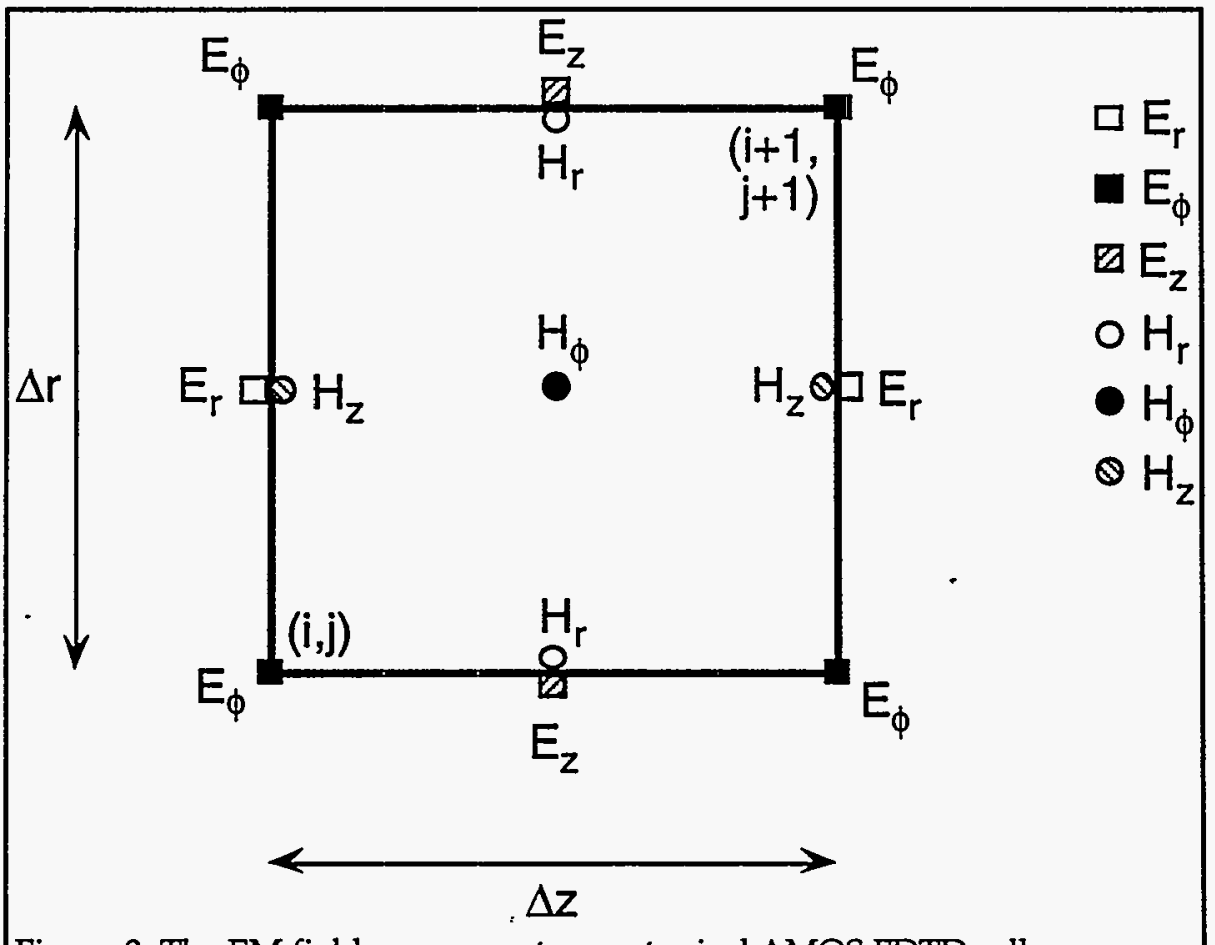

Figure 3. The EM field components on a typical AMOS FDTD cell. and $\mathrm{E}_{\phi}, \mathrm{H}_{\mathrm{r}}$, and $\mathrm{H}_{\mathbf{z}}$ vary as $\sin \phi$. The field distribution on a typical AMOS FDTD mesh element is shown in Fig. 3. The fields are distributed so that the Maxwell curl equations are center-differenced and the $\mathrm{E}$ and $\mathrm{H}$ fields are offset by a half time step $(\Delta t / 2)$ so that the time derivatives are also center-differenced. 


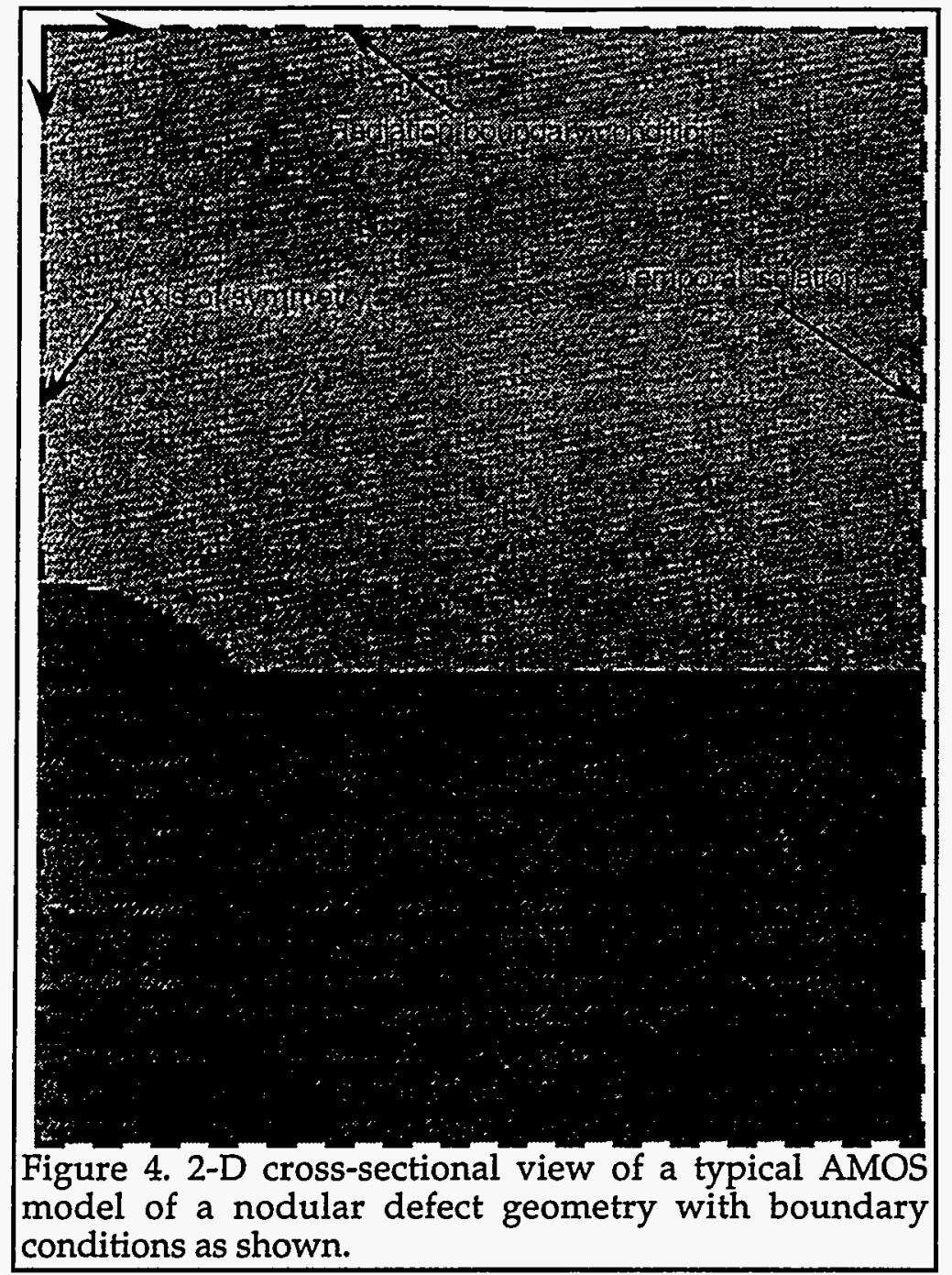

We developed a new output diagnostic for the AMOS solver, enabling us to obtain the single-frequency Fourier Transform coefficients of the electric field components at every node throughout a 2-D planar $r-z$ region. By relating the single-frequency Fourier Transform coefficients of the electric field components to the peak steady-state amplitudes of the electric field components, we were able to calculate the peak steady-state electric field everywhere within the defect geometry. (For a sinusoidal excitation, the peak steady-state electric field amplitude, $\mathrm{E}_{\mathrm{SS}}$, can be related to its single-frequency Fourier Transform coefficient, $\mathrm{E}_{\mathrm{fc}}$, by the relation $\mathrm{E}_{\mathrm{SS}}=2 \mathrm{Efc}_{\mathrm{f}}$ ) The peak steady-state electric field amplitude (in $\mathrm{V} / \mathrm{m}$ ) was then used to calculate the peak steady-state heat generation $\left(\sigma \mathrm{E}^{2}\right.$, in $\left.\mathrm{W} / \mathrm{m}^{3}\right)$ throughout the defect geometry.

Shown in Fig. 4 is a schematic of a 2-D r-z cross-section of a rotationally symmetric defect geometry showing the boundary conditions that were applied for the EM simulation. Fig. 5 shows an enlarged view of the 2-D AMOS mesh for a typical nodular defect. The AMOS computer model of the nodular defect uses a regular mesh consisting of 800,000 cells and whose 2-D r-z cross-section covers a region of approximately $45 \mu \mathrm{m}$ in the radial (r) direction and $9 \mu \mathrm{m}$ in the axial (z) direction with an individual square cell size of $d r=d z=0.022858 \mu \mathrm{m}$. This selection of cell size results in six cells per $\mathrm{HfO}_{2}$ quarter-wave layer thickness and eight cells per $\mathrm{SiO}_{2}$ quarter-wave layer thickness, as well as satisfying the 10-cells-per-wavelength rule-of-thumb for all materials in the model. The reason for the large radial extent of the geometry is to enforce the temporal isolation requirement in the radial direction (refer to Fig. 4). 


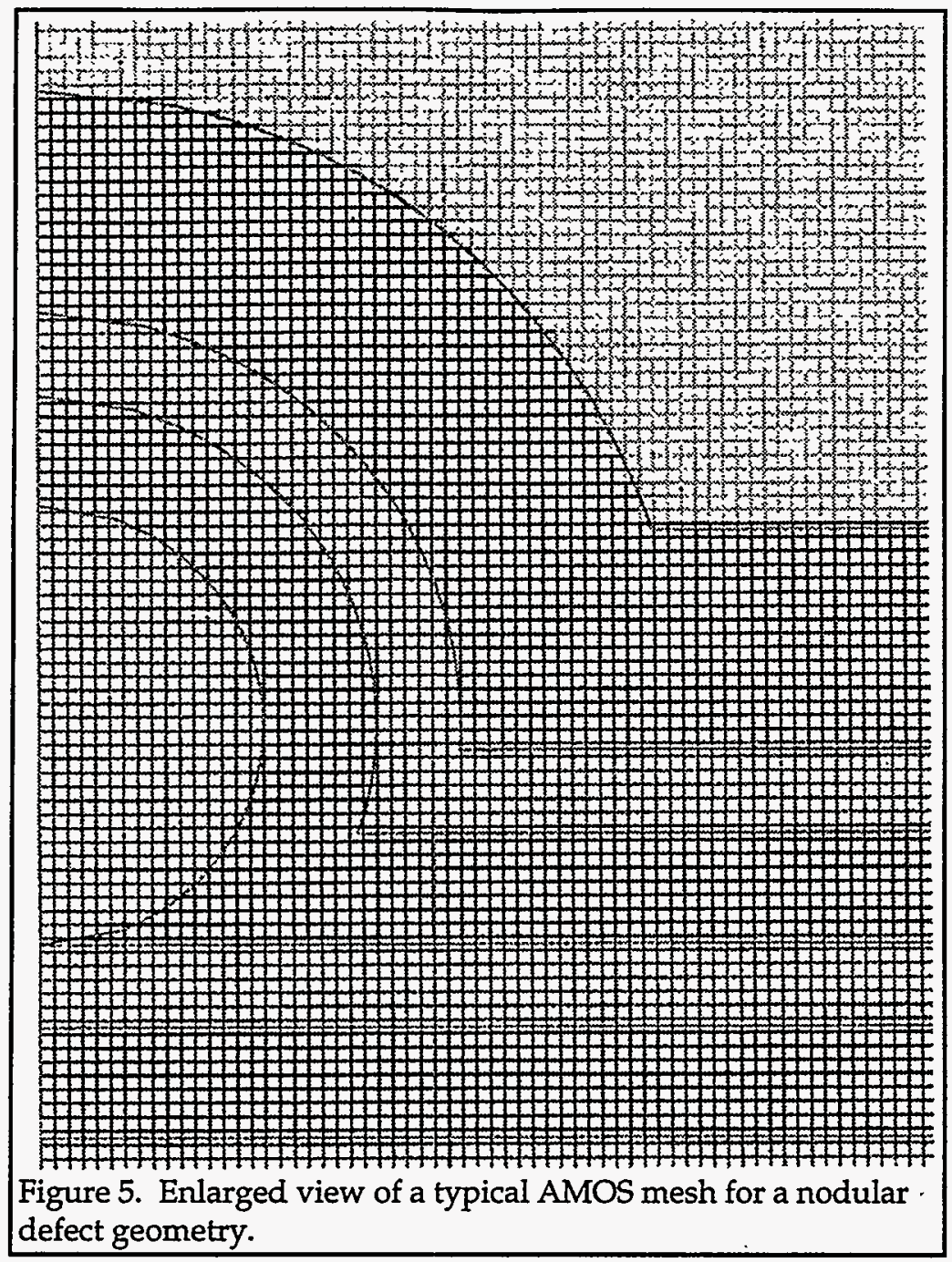

We used the 2-1/2-D FDTD EM code, AMOS, to perform computer simulations of 23 nodular defect geometries in order to investigate the effect of seed size, depth, and material composition on the electric field enhancement throughout the defect geometry. The results of these EM simulations will be discussed in the next section. These EM simulations were performed as one part of a more complex electro-mechanical computer modeling effort. Fig. 6 shows a block diagram of the electromechanical computer modeling effort. An EM simulation code is used to compute the peak steady-state electric fields everywhere throughout a defect geometry. The steady-state electric fields are then interpolated from the EM model mesh to the thermal-mechanical model mesh and the heat generation $\left(\sigma E^{2}\right)$ is computed at each cell in the thermal-mechanical model mesh. The heat generation terms are then used as the initial conditions for the thermal model which will compute the spatial and temporal distributions of temperature. These temperature profiles will then be used as inputs to the structural model which will compute the spatial and temporal distributions of stresses, thereby completing the electromechanical modeling process. This report will concentrate on the EM modeling portion of the electro-mechanical defect modeling effort. 


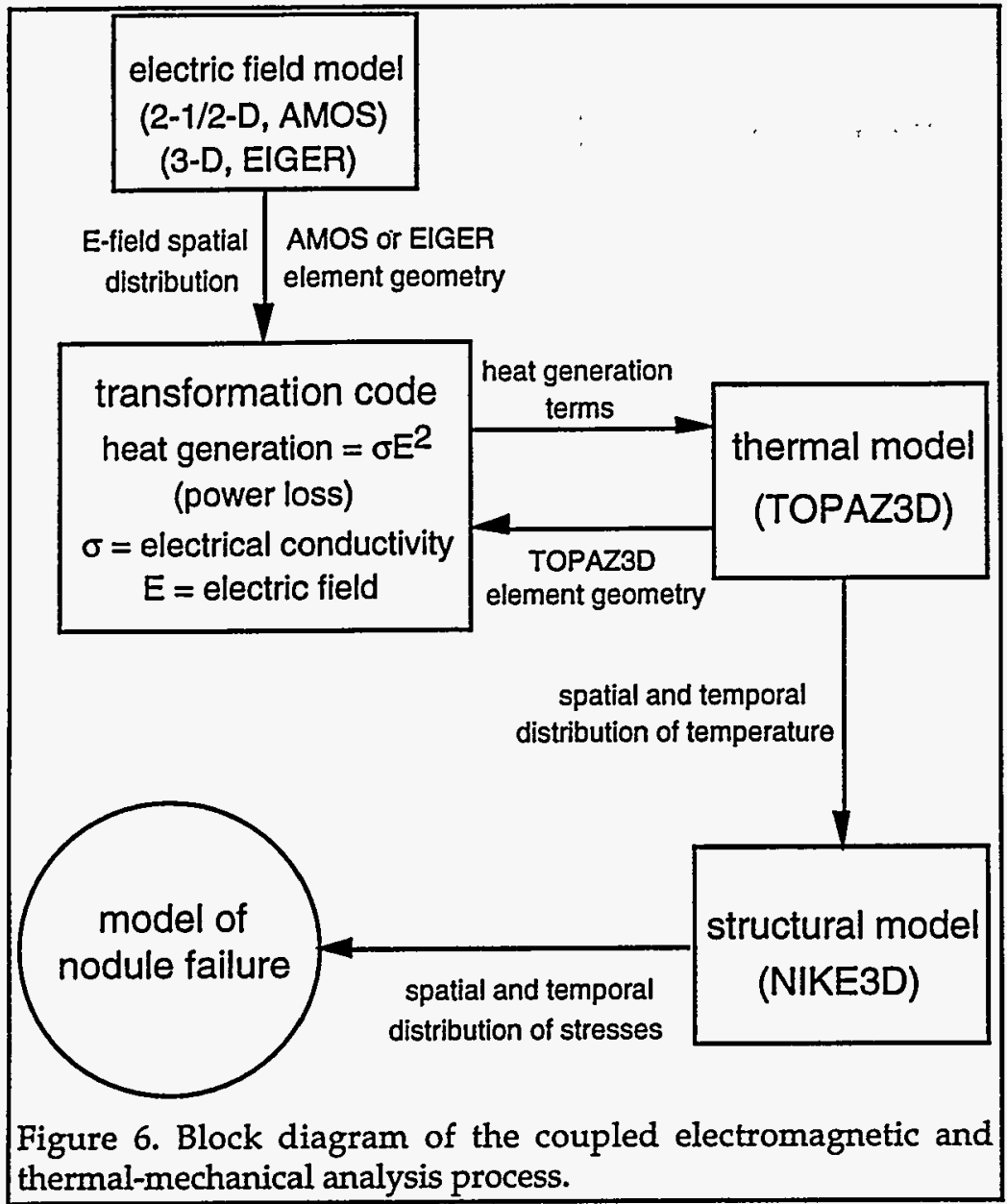

\section{Electromagnetic \\ Modeling Results}

A. Perfect HR Dielectric Stack

Prior to performing the 2-1/2-D FDTD EM analysis of the matrix of rotationallysymmetric defect geometries, the perfect dielectric multilayer $H R$ coating (in the absence of any defects) was modeled in order to verify the performance of the dielectric stack mirror as well as to generate a series of parametric design plots to assist in the selection of electrical properties such as the electrical conductivity and loss

tangent of each of the lowloss materials used in the model (i.e., $\mathrm{HfO}_{2}$ and $\mathrm{SiO}_{2}$ ) that are consistent with the experimentally determined power loss of $\mathrm{SiO}_{2} / \mathrm{HfO}_{2}$ HR coatings. Fig. 7 shows the steadystate electric field standing wave (EFSW) distribution (normalized to the input electric field)

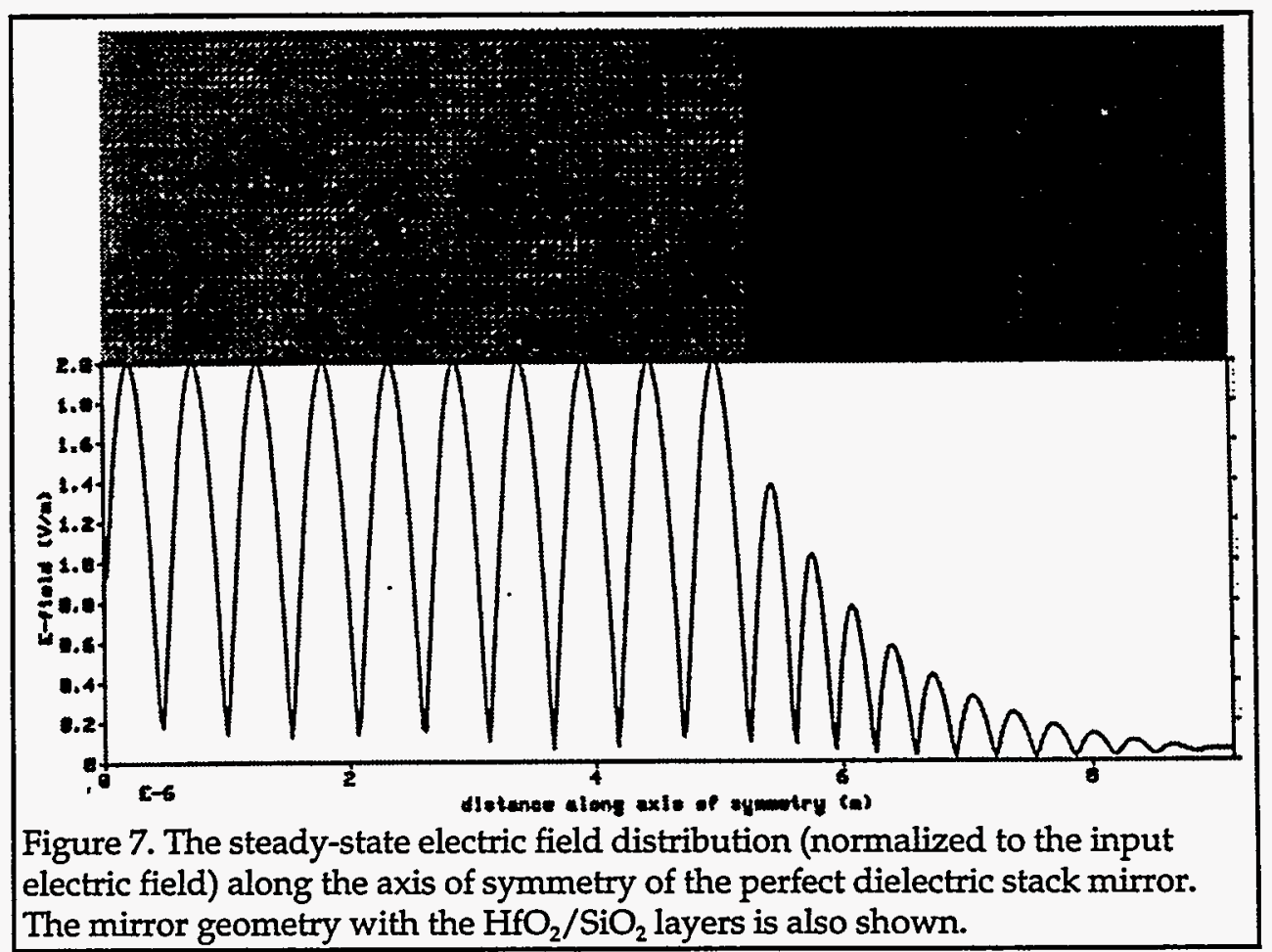




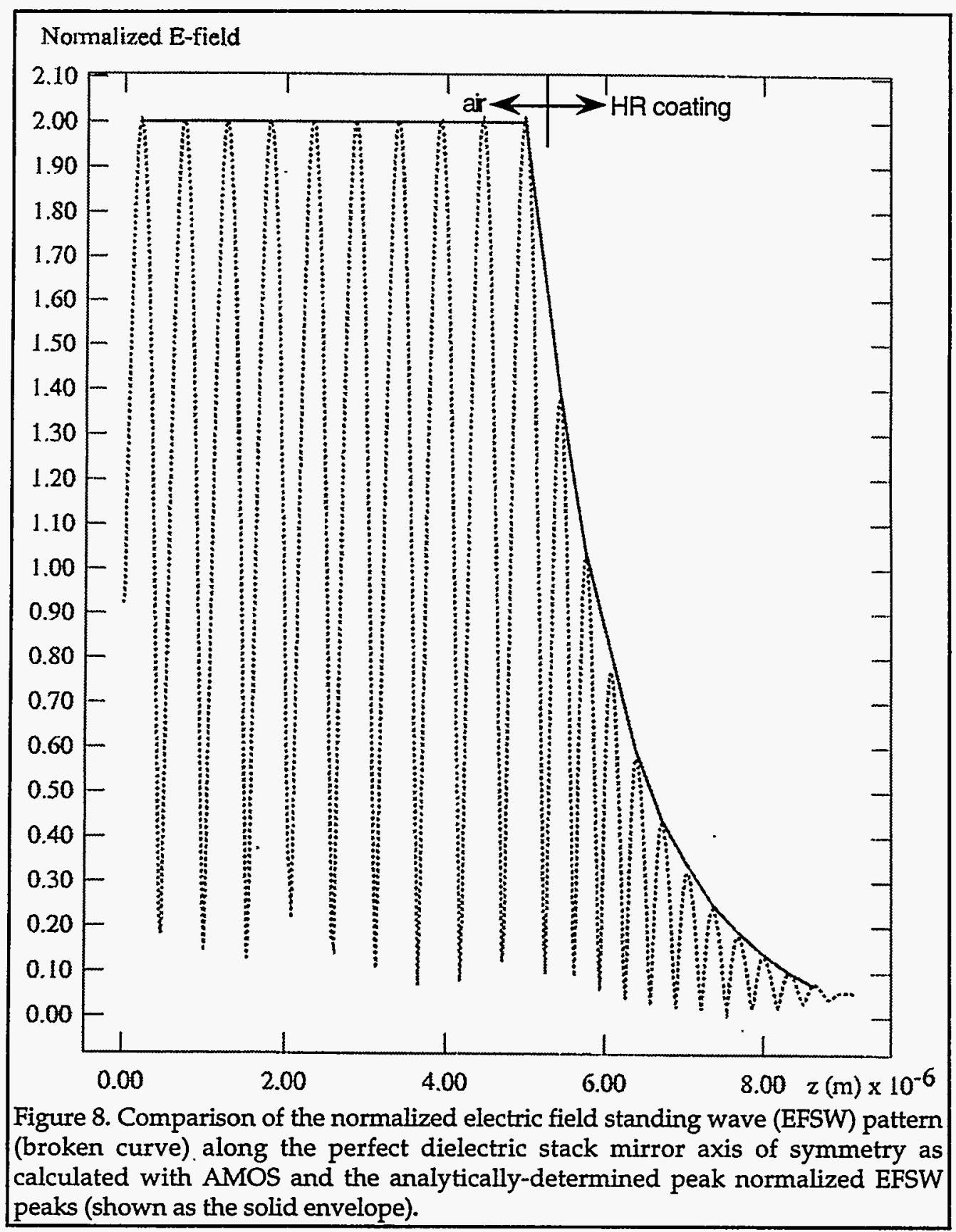

along the axis of symmetry of the perfect dielectric stack mirror. The mirror geometry with the $\mathrm{HfO}_{2} / \mathrm{SiO}_{2}$ layers is also shown. Fig. 7 demonstrates that the stack is, indeed, performing as an HR coating with the input electric field experiencing near-perfect reflection at the air/HR coating interface with an in-phase addition of the incoming and reflected electric fields, resulting in a peak normalized EFSW value of two in the air region in front of the multilayer dielectric stack. The EFSW pattern decays into the HR coating since most of the energy is reflected at the air/HR coating interface and, consequently, very little seeps into the HR coating. Fig. 8 compares the normalized EFSW pattern (broken curve) along the perfect dielectric stack mirror axis of symmetry as calculated with the AMOS code and the analytically-determined normalized EFSW peaks (shown as the solid envelope). 


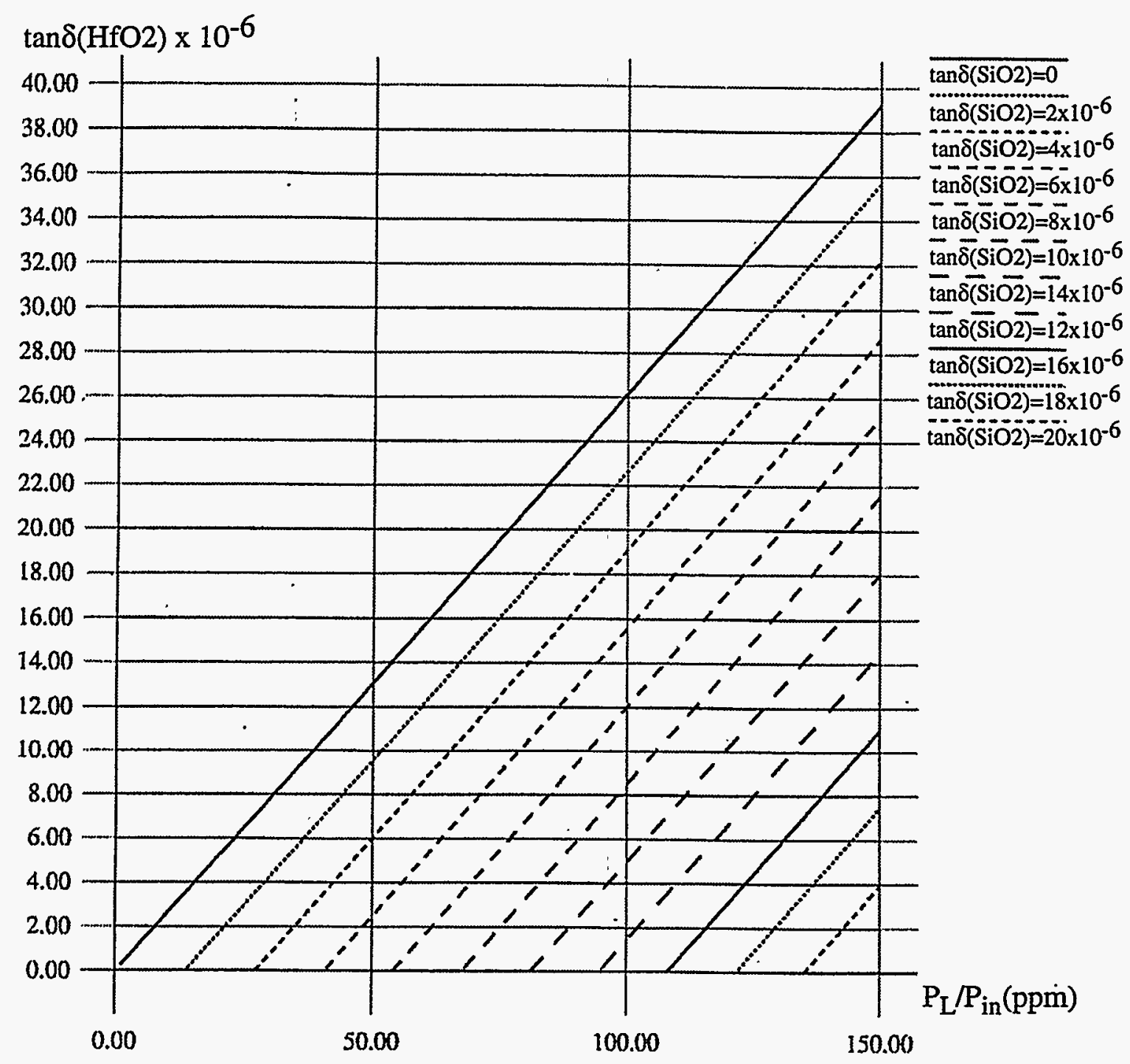

Figure 9. Parametric design plots showing the loss tangent of $\mathrm{HfO}_{2}$ as a function of power loss ratio (in parts-per-million, $\mathrm{ppm}$ ) in the coating (for several different values of loss tangent of $\mathrm{SiO}_{2}$ ).

The perfect dielectric stack mirror geometry was used to generate a series of parametric design plots showing the loss tangent $(\tan \delta)$ of $\mathrm{HfO}_{2}$ as a function of the power loss ratio (in parts-per-million, $\mathrm{ppm}$ ) of the HR coating for several different values of loss tangent of $\mathrm{SiO}_{2}$ as depicted in Fig. 9. The parametric plots in Fig. 9 show that there are an infinite number of possible loss tangent pairs (for $\mathrm{HfO}_{2}$ and $\mathrm{SiO}_{2}$ ) for each value of power loss ratio. Therefore, in addition to a value for the power loss ratio, a second piece of information concerning the electrical properties of the materials is required in order to narrow the infinite choice of loss tangent pairs down to a unique pair. Although experimental measurements of the loss tangents for thin films of hafnia 
and silica at optical frequencies have not been reported in the literature, the literature does generally assume hafnia to be the lossier of the two materials. Therefore, we have assumed a reasonable value for the loss tangent ratio of hafnia to silica to be: $\tan \delta\left(\mathrm{HfO}_{2}\right) / \tan \delta\left(\mathrm{SiO}_{2}\right)=2$. Based on this assumption and given a typical measured value of power loss ratio to be $\sim 150 \mathrm{ppm}$ for the $\mathrm{HfO}_{2} / \mathrm{SiO}_{2} \mathrm{HR}$ coatings, the infinite choice for loss tangents was narrowed down to a unique choice of $10.2 \times 10^{-6}$ for $\mathrm{SiO}_{2}$ and $20.4 \times 10^{-6}$ for $\mathrm{HfO}_{2}$, or equivalently, down to a unique choice of electrical conductivities of $0.3366 \mathrm{~S} / \mathrm{m}$ for $\mathrm{SiO}_{2}$ and $1.1956 \mathrm{~S} / \mathrm{m}$ for $\mathrm{HfO}_{2}$ (for an optical wavelength of $1.06 \mu \mathrm{m})$. As mentioned previously, the loss tangent $(\tan \delta)$ and electrical conductivity $(\sigma)$ are related by the following equation: $\tan \delta=\sigma / \omega \varepsilon$, with $\omega$, the radial frequency ( $\omega=2 \pi \mathrm{f}$, with $\mathrm{f}$, the optical frequency in $\mathrm{Hz}$ ) and $\varepsilon=\varepsilon_{\mathrm{r}} * \varepsilon_{0}$, with $\varepsilon_{0}=8.85 \mathrm{x}$ $10^{-12} \mathrm{~F} / \mathrm{m}$, the permittivity of free space and $\varepsilon_{\mathrm{r}}$, the relative permittivity.

\section{B. Nodular Defect Geometries}

A 2-1/2-D FDTD EM analysis was performed on the matrix of 23 rotationallysymmetric defect geometries shown in Table 2. (Refer to Fig. 2 for a schematic of a typical nodular defect geometry.) In all geometries studied, a 23-layer $\mathrm{HfO}_{2} / \mathrm{SiO}_{2} \mathrm{HR}$ multilayer coating was assumed as the basic underlying geometry with a seed of diameter, $d$, embedded at some depth, $T$, within the 23-layer HR coating. The matrix of 23 geometries shown in Table 2 was selected in order to study the effects on the electric field enhancement of varying the seed diameter, seed depth, and seed material. All geometries assumed a $\mathrm{HfO}_{2}$ seed, except for geometry \#6 which assumed a $\mathrm{SiO}_{2}$ seed.

Because the embedded seed perturbs the HR coating geometry, this coating no longer looks like a perfect mirror and will therefore no longer exhibit the EFSW pattern that was shown in Fig. 7. Rather than exhibiting the simple decay of the EFSW pattern within the HR coating that was shown in Fig. 7 for the perfect mirror, the EFSW pattern along the axis of symmetry of the nodular defect consists of a complex series of local electric field enhancements whose location and magnitude depend on the defect geometry. These electric field enhancements may be due to a complex combination of higher-order EM effects, including focusing and resonance effects. 


\begin{tabular}{|c|c|c|c|c|c|}
\hline geom \# & seed material & $\mathrm{d}[\mu \mathrm{m}]$ & $\mathrm{D}[\mu \mathrm{m}]$ & $\mathrm{T}[\mu \mathrm{m}]$ & T [\# layers] \\
\hline 1 & $\mathrm{HfO}_{2}$ & 0.73 & 3.392 & 1.97 & 11 \\
\hline 2 & $\mathrm{HfO}_{2}$ & 0.25 & 1.985 & 1.97 & 11 \\
\hline 3 & $\mathrm{HfO}_{2}$ & 1.50 & 4.862 & 1.97 & 11 \\
\hline 4 & . $\mathrm{HfO}_{2}$ & 0.73 & 2.417 & 1.00 & 5 \\
\hline 5 & $\mathrm{HfO}_{2}$ & 0.73 & 4.144 & 2.94 & 17 \\
\hline 6 & $\mathrm{SiO}_{2}$ & 0.73 & 3.392 & 1.97 & 11 \\
\hline 7 & $\mathrm{HfO}_{2}$ & 0.10 & 1.255 & 1.97 & 11 \\
\hline 8 & $\mathrm{HfO}_{2}$ & 1.09769 & 4.159 & 1.97 & 11 \\
\hline 9 & $\mathrm{HfO}_{2}$ & 0.73 & 2.007 & 0.69 & 3 \\
\hline 10 & $\mathrm{HfO}_{2}$ & 1.50 & 3.464 & 1.00 & 5 \\
\hline 11 & $\mathrm{HfO}_{2}$ & 1.50 & .5 .940 & 2.94 & 17 \\
\hline 12 & $\mathrm{HfO}_{2}$ & 0.25 & 2.425 & 2.94 & 17 \\
\hline 13 & $\mathrm{HfO}_{2}$ & 1.09769 & 2.963 & 1.00 & 5 \\
\hline 14 & $\mathrm{HfO}_{2}$ & 0.55 & 2.944 & 1.97 & 11 \\
\hline 15 & $\mathrm{HfO}_{2}$ & 0.90 & 3.766 & 1.97 & 11 \\
\hline 16 & $\mathrm{HfO}_{2}$ & 1.09769 & 2.963 & 1.00 & 5 \\
\hline 17 & $\mathrm{HfO}_{2}$ & 0.90 & 2.683 & 1.00 & 5 \\
\hline 18 & $\mathrm{HfO}_{2}$ & 1.09769 & 5.081 & 2.94 & 17 \\
\hline 19 & $\mathrm{HfO}_{2}$ & 0.90 & 4.601 & 2.94 & 17 \\
\hline 20 & $\mathrm{HfO}_{2}$ & 0.25 & 1.414 & 1.00 & 5 \\
\hline 21 & $\mathrm{HfO}_{2}$ & 1.09769 & 2.462 & 0.69 & 3 \\
\hline 22 & $\mathrm{HfO}_{2}$ & 0.6761 & 3.264 & 1.97 & 11 \\
\hline 23 & $\mathrm{HfO}_{2}$ & 0.7849 & 3.517 & 1.97 & 11 \\
\hline
\end{tabular}

Table 2. Table of the 23 nodular defect geometries that were studied with $\mathrm{d}$, the seed diameter; $\mathrm{D}$, the defect dome diameter; and T, the seed depth (refer to schematic in Fig. 2).

Fig. 10 shows the steady-state electric field profile everywhere within a 2-D r-z cross-section (for $\phi=0^{\circ}$ ) of a typical nodular defect geometry. In this case, the nodular defect geometry consists of a spherical $\mathrm{HfO}_{2}$ seed of diameter, $\mathrm{d}=0.73 \mu \mathrm{m}$; depth, $\mathrm{T}=$ $1.97 \mu \mathrm{m}$; and nodule dome diameter of $\mathrm{D}=3.392 \mu \mathrm{m}$. The source was assumed to be a normally-incident, sinusoidally-varying, linearly-polarized, plane wave $\left(\lambda_{\mathrm{o}}=1.06 \mu \mathrm{m}\right)$ with an average input power density of $1 \mathrm{GW} / \mathrm{cm}^{2}$. As is illustrated in Fig. 10, for rotationally-symmetric nodular defect geometries under normal-incidence illumination, the peak electric fields and highest electric field enhancements are typically located along the axis of symmetry. The EFSW pattern along the axis of symmetry $(r=0 \mu \mathrm{m})$ of the nodule as well as the EFSW pattern along a line parallel to the axis of symmetry but at a radius of $5 \mu \mathrm{m}$ are also shown in Fig. 10. The EFSW pattern along the axis of symmetry shows several localized electric field enhancements within the HR coating, one in the $\mathrm{SiO}_{2}$ overcoat and one in the $\mathrm{HfO}_{2}$ seed, while the EFSW pattern for the line 


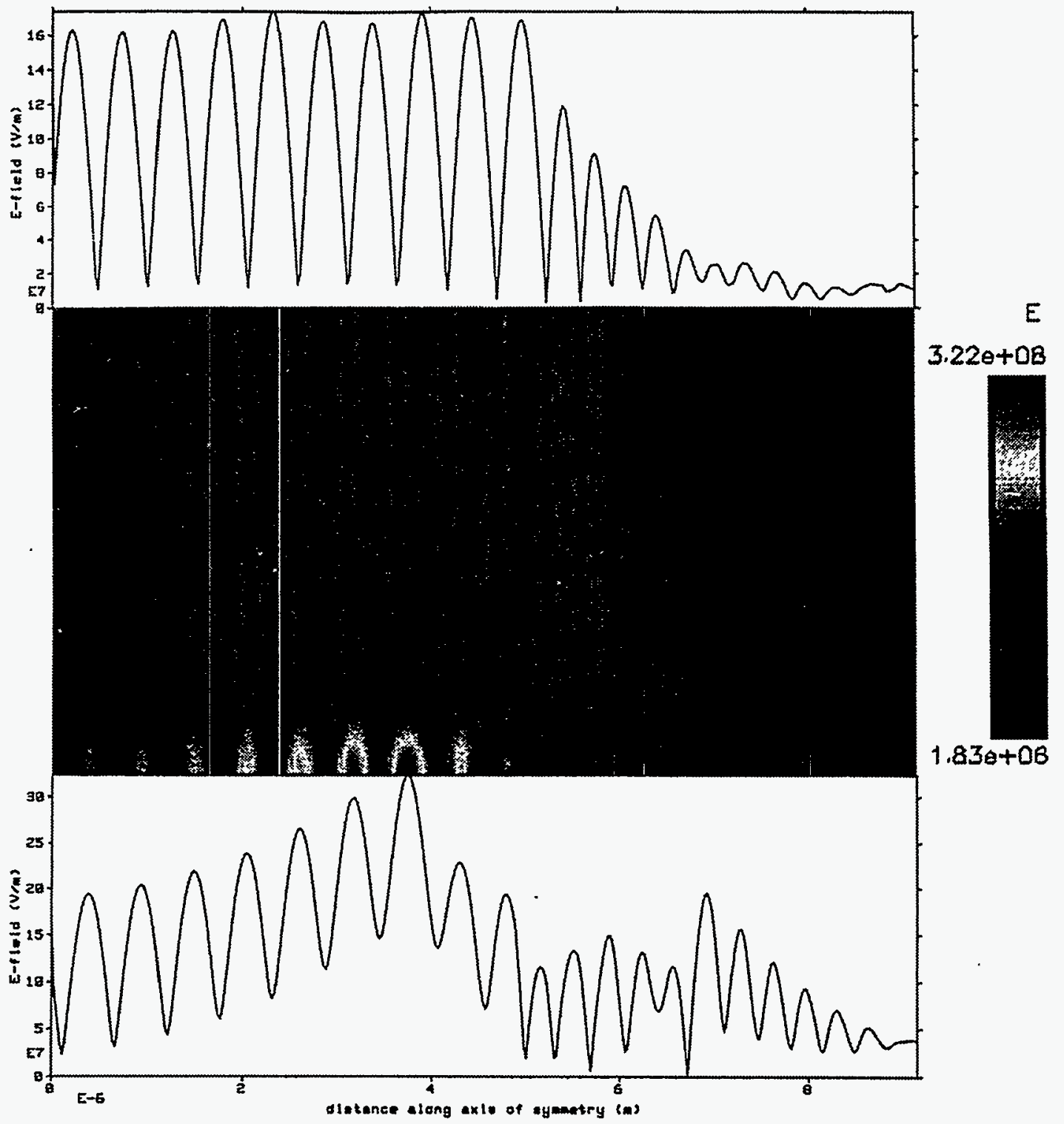

Figure 10. The steady-state electric field profile everywhere within a 2-D r-z cross-section (for $\phi=0^{\circ}$ ) of a typical nodular defect geometry, consisting of a spherical $\mathrm{HfO}_{2}$ seed of diameter, $\mathrm{d}=0.73 \mu \mathrm{m}$ and depth, $\mathrm{T}=1.97 \mu \mathrm{m}$. Also shown is the EFSW pattern along the axis of symmetry $(\mathrm{r}=0 \mu \mathrm{m})$ of the nodule as well as the EFSW pattern along a line parallel to the axis of symmetry but at a radius of $5 \mu \mathrm{m}$.

at $\mathrm{r}=5 \mu \mathrm{m}$ looks very much like that of the perfect HR stack. This is to be expected since for radii larger than the defect dome radius, $D / 2=1.696 \mu \mathrm{m}$, the nodule defect geometry rapidly returns to the perfect stack geometry. By comparing the EFSW pattern along the axis of symmetry to that along the line at $r=5 \mu \mathrm{m}$ (i.e., to the EFSW pattern of a perfect HR stack), we see that the peak electric field enhancement for this particular geometry is approximately a factor of 2.2. This peak electric field enhancement is 
determined by comparing the peak electric field in the lossier material (i.e., in one of the $\mathrm{HfO}_{2}$ quarter-wave layers or in the $\mathrm{HfO}_{2}$ seed) in the nodule defect geometry to the peak electric field in the lossier material (i.e., in the first $\mathrm{HfO}_{2}$ quarter-wave layer) in the perfect HR stack.

Typically, the electric field enhancements in the lossier material (i.e., in the $\mathrm{HfO}_{2}$ layers) are of the most importance since they will lead to "hot spots" in the heat generation $\left(\sigma \mathrm{E}^{2}\right)$ distribution and will therefore control the damage threshold of the HR coating. Figs. 11(a); (b), and (c) show the heat generation distributions (in $W / \mu \mathrm{m}^{3}$ ) that correspond to the electric field profile shown in Fig. 10, for three different choices of loss tangent ratios: (1) $\tan \delta\left(\mathrm{HfO}_{2}\right) / \tan \delta\left(\mathrm{SiO}_{2}\right)=2, \sigma_{\mathrm{HfO}_{2}}=$ of $1.1956 \mathrm{~S} / \mathrm{m}$ and, $\sigma_{\mathrm{SiO}_{2}}=0.3366$ $\mathrm{S} / \mathrm{m},(2) \tan \delta\left(\mathrm{HfO}_{2}\right) / \tan \delta\left(\mathrm{SiO}_{2}\right)=1, \sigma_{\mathrm{HfO}_{2}}=$ of $0.8138 \mathrm{~S} / \mathrm{m}$ and, $\sigma_{\mathrm{SiO}_{2}}=0.4582 \mathrm{~S} / \mathrm{m}$, and (3) $\tan \delta\left(\mathrm{HfO}_{2}\right) / \tan \delta\left(\mathrm{SiO}_{2}\right)=0.5, \sigma_{\mathrm{HFO}_{2}}=$ of $1.914 \mathrm{~S} / \mathrm{m}$ and, $\sigma_{\mathrm{SiO}_{2}}=0.1078 \mathrm{~S} / \mathrm{m}$. The choice of conductivities depicted in the heat generation plot in Fig. 11(a), corresponding to $\tan \delta\left(\mathrm{HfO}_{2}\right) / \tan \delta\left(\mathrm{SiO}_{2}\right)=2$ (resulting in $\sigma_{\mathrm{HfO}_{2}}=$ of $1.1956 \mathrm{~S} / \mathrm{m}$ and, $\sigma_{\mathrm{SiO}_{2}}=0.3366 \mathrm{~S} / \mathrm{m}$ ), was the choice that was used for all heat generation calculations for the 23 geometries that were studied. However, the additional two cases of loss tangent ratio whose heat generation profiles are also shown in Fig. 11 demonstrate the importance of selecting a realistic value of loss tangent ratio for the materials that comprise the HR coating. Since the heat generation is calculated as $\sigma \mathrm{E}^{2}$, it is evident that the peaks in the electric field profile that lie in the lossier material (the material with the higher loss tangent, $\tan \delta$, and the higher electrical conductivity, $\sigma$ ) will be emphasized in the heat generation profile while the electric field peaks in the less lossy material will be de-emphasized in the heat generation profile.

Fig. 12 shows the steady-state electric field profile everywhere within a 2-D r-z cross-section (for $\phi=0^{\circ}$ ) of a nodular defect geometry containing a large $\mathrm{HfO}_{2}$ seed of diameter, $\mathrm{d}=1.5 \mu \mathrm{m}$; and shallow depth, $\mathrm{T}=1 \mu \mathrm{m}$. Also shown in Fig. 12 is the EFSW pattern along the axis of symmetry $(\mathrm{r}=0 \mu \mathrm{m})$. This nodular defect geometry exhibited an electric field enhancement of 6.4, the largest of all the geometries studied. Fig. 13 shows the corresponding heat generation $\left(\sigma E^{2}\right)$ plot. Note that an electric field enhancement of 6.4 would lead to an enhancement in the heat generation $\left(\sigma E^{2}\right)$ of a factor of 41 . Fig. 14 shows the steady-state electric field profile everywhere within a 2-D $\mathrm{r}-\mathrm{z}$ cross-section (for $\phi=0^{\circ}$ ) of a nodular defect geometry containing a small $\mathrm{HfO}_{2}$ seed of diameter, $\mathrm{d}=0.25 \mu \mathrm{m}$; and large depth, $\mathrm{T}=2.94 \mu \mathrm{m}$, with the EFSW pattern along the axis of symmetry $(\mathrm{r}=0 \mu \mathrm{m})$ also shown. In this case, the electric field enhancement of 1.3 was the smallest of all the geometries studied. Fig. 15 shows the corresponding heat 


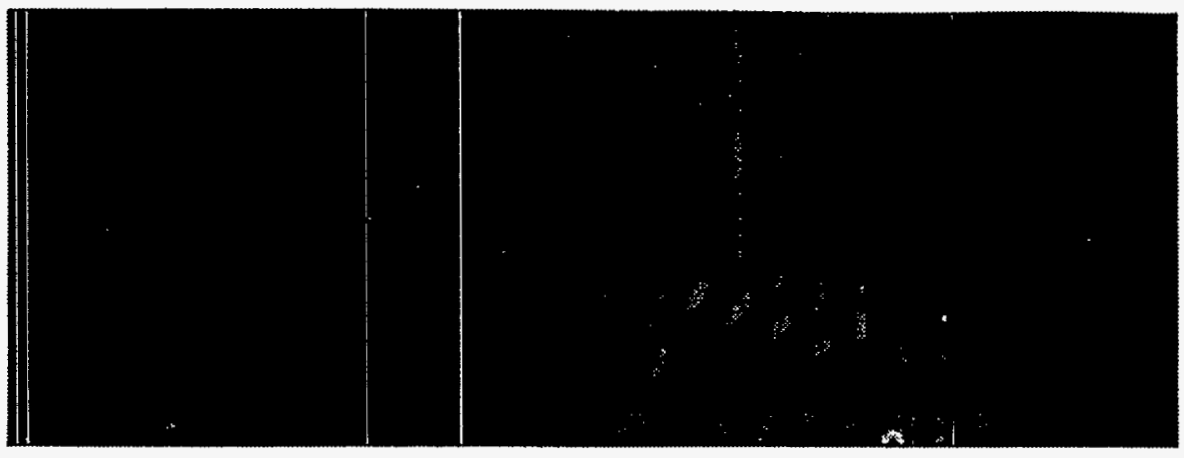

a.

b.

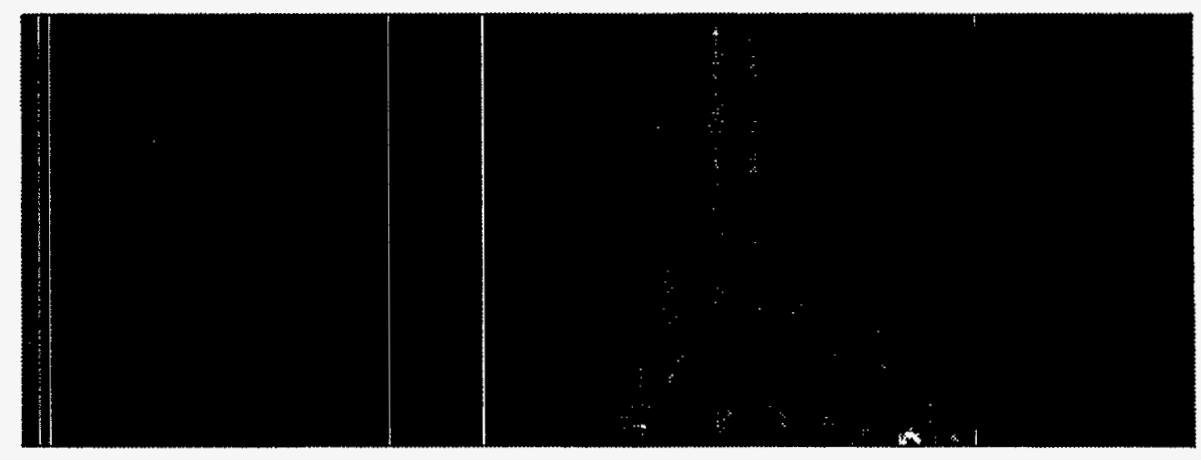

$3.04 \theta-02$

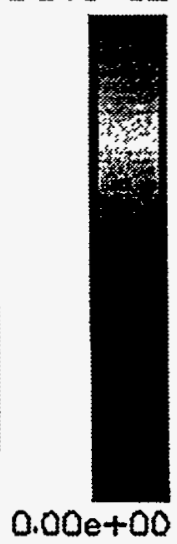

$\mathrm{s} * \mathrm{E} 2$

$2.13 e-02$
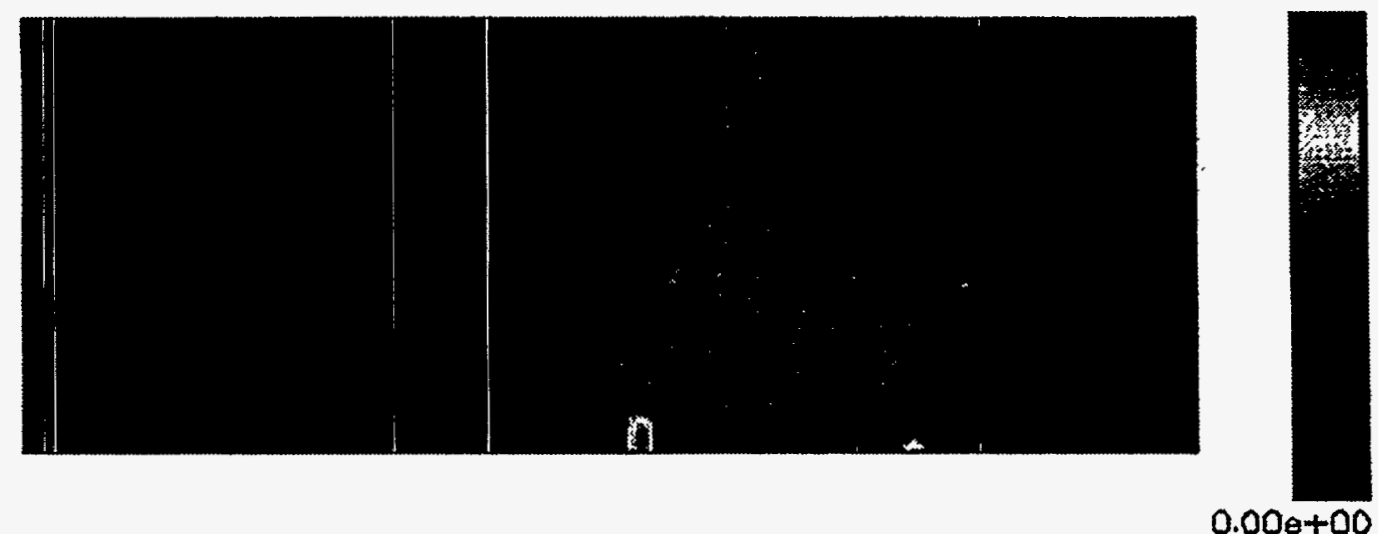

Figure 11. The heat generation distribution $\left(\sigma \mathrm{E}^{2}\right.$ in $\left.\mathrm{W} / \mu \mathrm{m}^{3}\right)$ that corresponds to the electric field profile shown in Fig. 10, for three different choices of loss tangent ratios:

(a) $\tan \delta\left(\mathrm{HfO}_{2}\right) / \tan \delta\left(\mathrm{SiO}_{2}\right)=2, \sigma_{\mathrm{HFO}}=$ of $1.1956 \mathrm{~S} / \mathrm{m}$ and , $\sigma_{\mathrm{SiO} 2}=0.3366 \mathrm{~S} / \mathrm{m}$,

(b) $\tan \delta\left(\mathrm{HfO}_{2}\right) / \tan \delta\left(\mathrm{SiO}_{2}\right)=1, \sigma_{\mathrm{HFO} 2}=$ of $0.8138 \mathrm{~S} / \mathrm{m}$ and,$\sigma_{\mathrm{SiO} 2}=0.4582 \mathrm{~S} / \mathrm{m}$, and

(c) $\tan \delta\left(\mathrm{HfO}_{2}\right) / \tan \delta\left(\mathrm{SiO}_{2}\right)=0.5, \sigma_{\mathrm{HrO} 2}=$ of $1.914 \mathrm{~S} / \mathrm{m}$ and,$\sigma_{\mathrm{SiO} 2}=0.1078 \mathrm{~S} / \mathrm{m}$. 


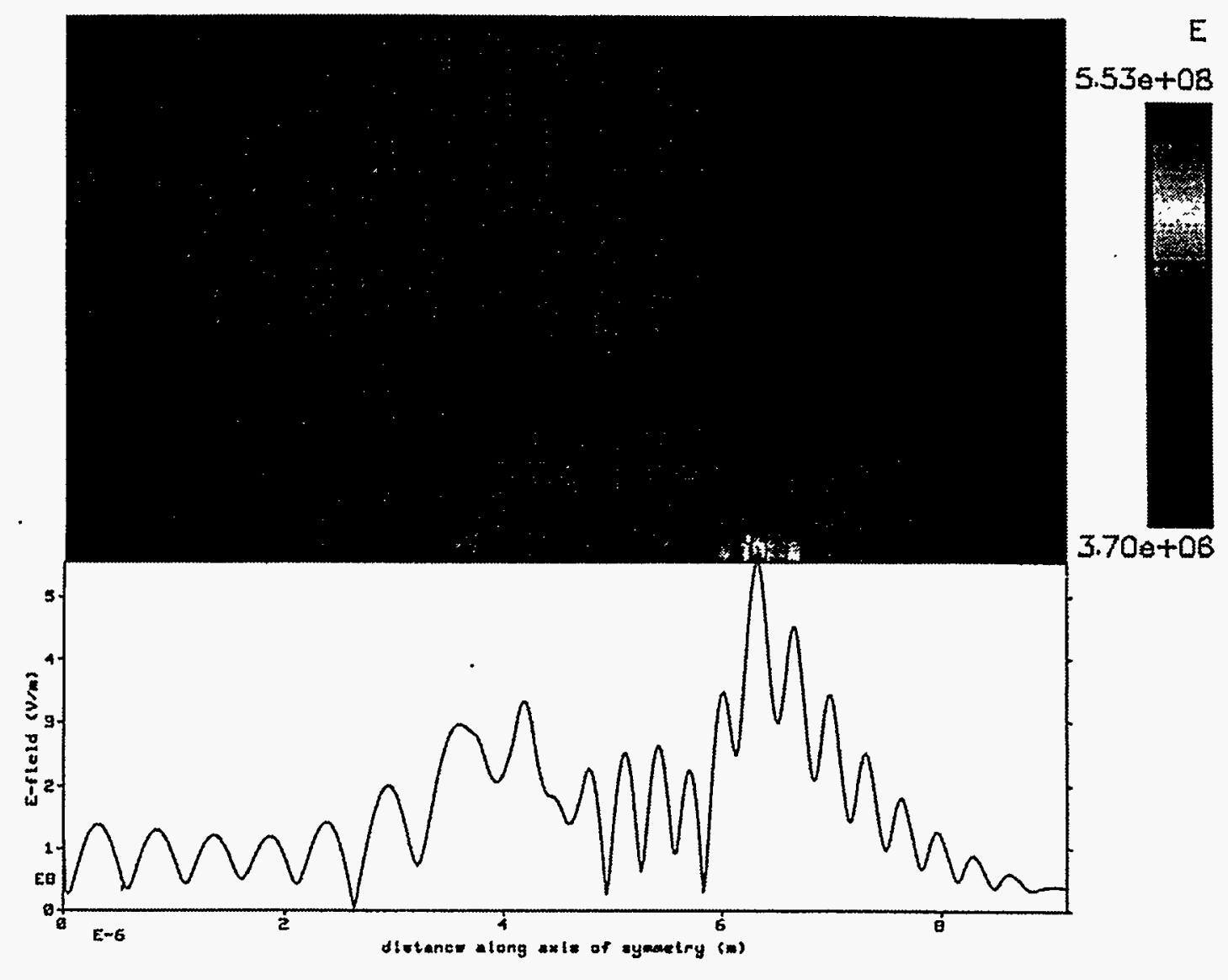

Figure 12. The steady-state electric field profile everywhere within a 2-D r-z cross-section (for $\phi=0^{\circ}$ ) of a nodular defect geometry containing a large $\mathrm{HfO}_{2}$ seed of diameter, $\mathrm{d}=1.5 \mu \mathrm{m}$; and shallow depth, $\mathrm{T}=1$ $\mu \mathrm{m}$. Also shown is the EFSW pattern along the axis of symmetry $(r=0 \mu \mathrm{m})$.

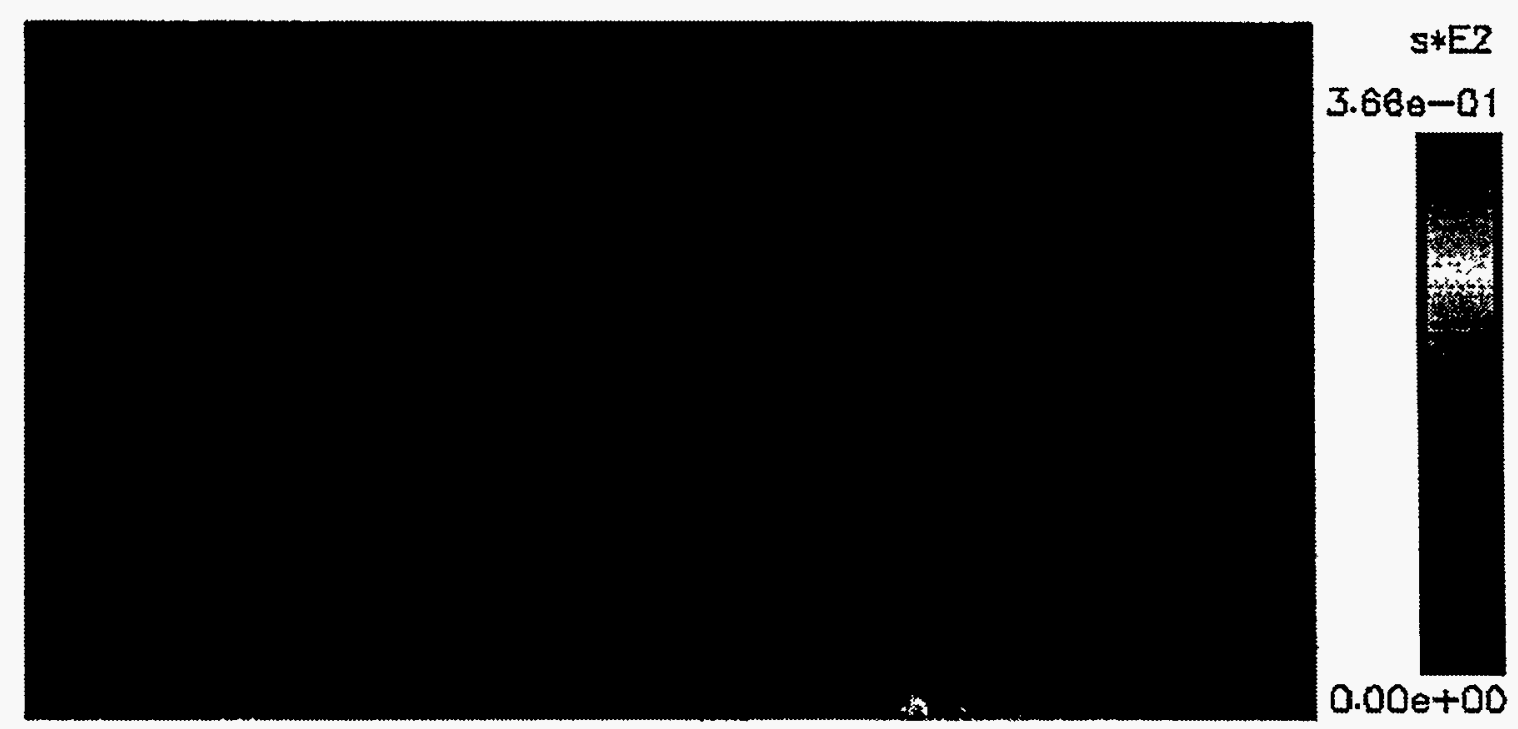

Figure 13. The heat generation distribution $\left(\sigma \mathrm{E}^{2}\right.$ in $\left.\mathrm{W} / \mu \mathrm{m}^{3}\right)$ that corresponds to the electric field profile shown in Fig. 12. 


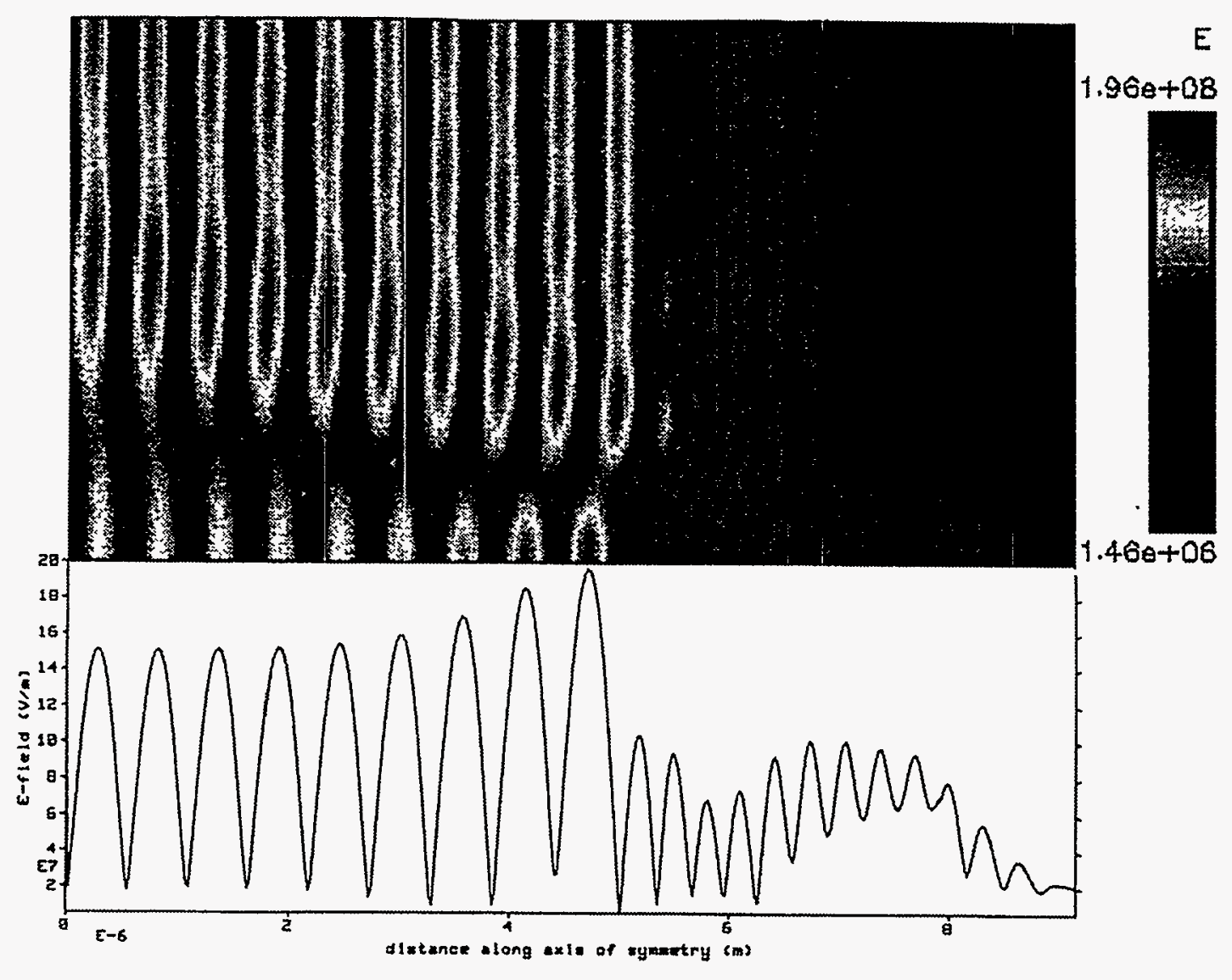

Figure 14. The steady-state electric field profile everywhere within a 2-D r-z cross-section (for $\phi=0^{\circ}$ ) of a nodular defect geometry containing a small $\mathrm{HfO}_{2}$ seed of diameter, $\mathrm{d}=0.25 \mu \mathrm{m}$; and large depth, $\mathrm{T}=2.94$ $\mu \mathrm{m}$, with the EFSW pattern along the axis of symmetry $(\mathrm{r}=0 \mu \mathrm{m})$ also shown.

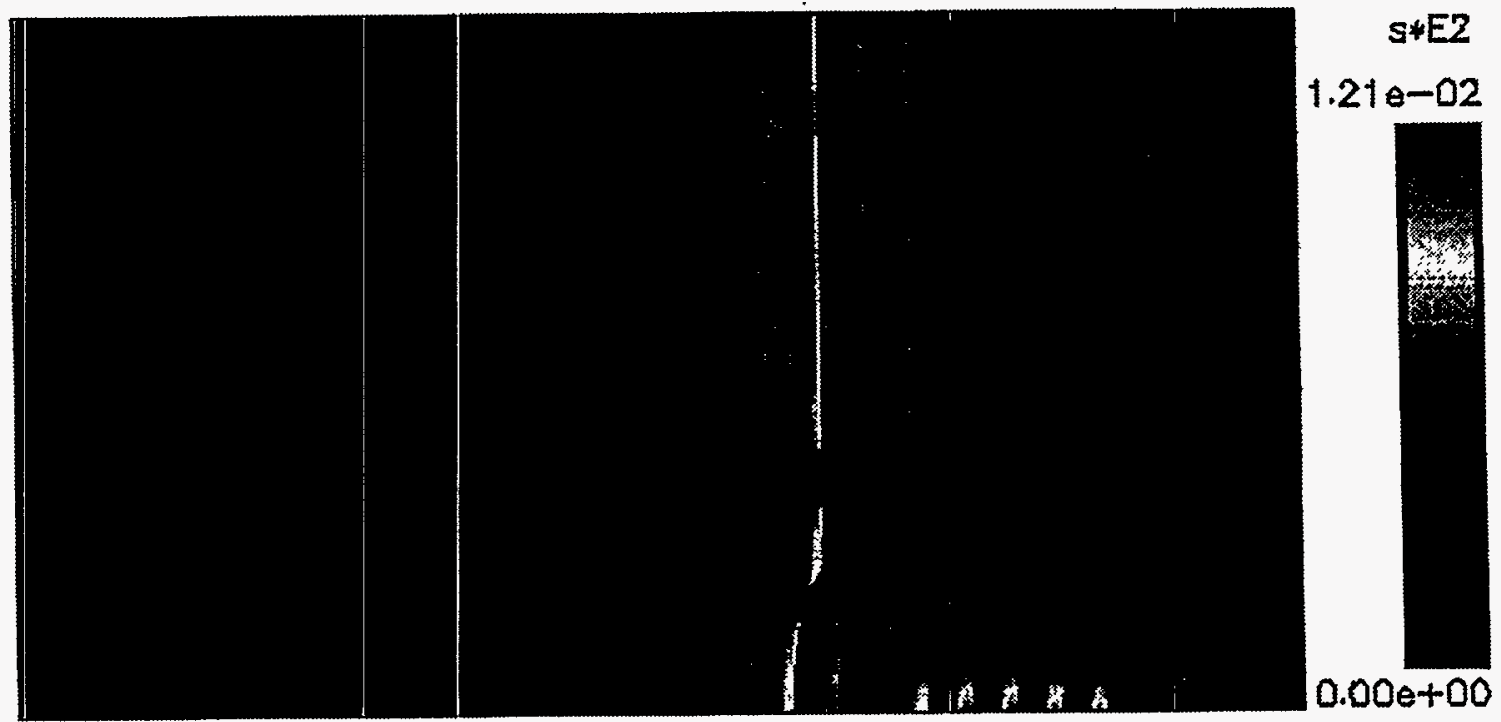

Figure 15. The heat generation distribution $\left(\sigma E^{2}\right.$ in $\left.W / \mu \mathrm{m}^{3}\right)$ that corresponds to the electric field profile shown in Fig. 14. 
generation $\left(\sigma \mathrm{E}^{2}\right.$ in $\left.\mathrm{W} / \mu \mathrm{m}^{3}\right)$ plot. These results predict that, as a general rule-of-thumb, geometries containing large, shallow seeds lead to the largest electric-field enhancements (or, equivalently, to the highest heat generation) while geometries containing small, deep seeds lead to the smallest electric field enhancements (or, equivalently, to the lowest heat generation).

However, the dependence of the electric field distribution, the electric field enhancement, and the heat generation on the nodular defect geometry (e.g., seed diameter, seed depth, and seed material composition) is, in general, quite complex. Variations in the nodular defect geometry not only affect the peak value of the electric field in the geometry, but also the electric-field profile itself. As the geometry parameters are varied, the locations of the "hot spots" move around within the geometry. For example, as the seed depth is varied for the medium seed $(\mathrm{d}=0.73 \mu \mathrm{m})$, the peak electric field value lies in the $\mathrm{HfO}_{2}$ defect for seed depths of $\mathrm{T}=0.69 \mu \mathrm{m}$ and $\mathrm{T}$ $=1.97 \mu \mathrm{m}$, but lies in $\mathrm{a} \mathrm{SiO}_{2}$ layer for seed depths of $\mathrm{T}=1.00 \mu \mathrm{m}$ and $\mathrm{T}=2.94 \mu \mathrm{m}$.

Although precise trends have been somewhat difficult to identify for all geometries studied, some trends have been observed in the EM modeling data. For example, it has been observed that for smaller seeds ( $d \leq 1 \mu \mathrm{m})$ the shallower the seed, the higher the peak electric field, electric-field enhancement, and heat generation, as shown in Figs. 16, 17, and 18, respectively. This indicates that for small seeds, the seed depth is the important factor affecting the electric field enhancement. For larger seed sizes, on the other hand, the dependence on seed depth is more complex and no such clear trend exists.

Figs. 19 and 20 show the envelopes of the normalized EFSW pattern (normalized to the input electric field) along the axis of symmetry $(\mathrm{r}=0 \mu \mathrm{m})$ at three different seed depths for the small $(\mathrm{d}=0.25 \mu \mathrm{m})$ and medium $(\mathrm{d}=0.73 \mu \mathrm{m})$ seeds, respectively. The air-HR interface is indicated in each figure as is the location of the seed (the solid gray line segment) for each curve. It is apparent from Fig. 19 that for the small seed, the "hot spots" in the electric field profile move around relative to the seed location for the different seed depths. The peak value of the electric field decreases as the seed depth is increased. In addition, the peaking in the electric field out in the air region remains virtually constant in amplitude and location as the seed depth is varied. Fig. 20 shows that, for the medium seed, the "hot spots" in the electric field profile are located within the seed for the shallower depths, but that the "hot spots" move to the $\mathrm{HfO}_{2}$ and $\mathrm{SiO}_{2}$ layers near the air-HR interface for the deeply embedded seed. The peak value of the 


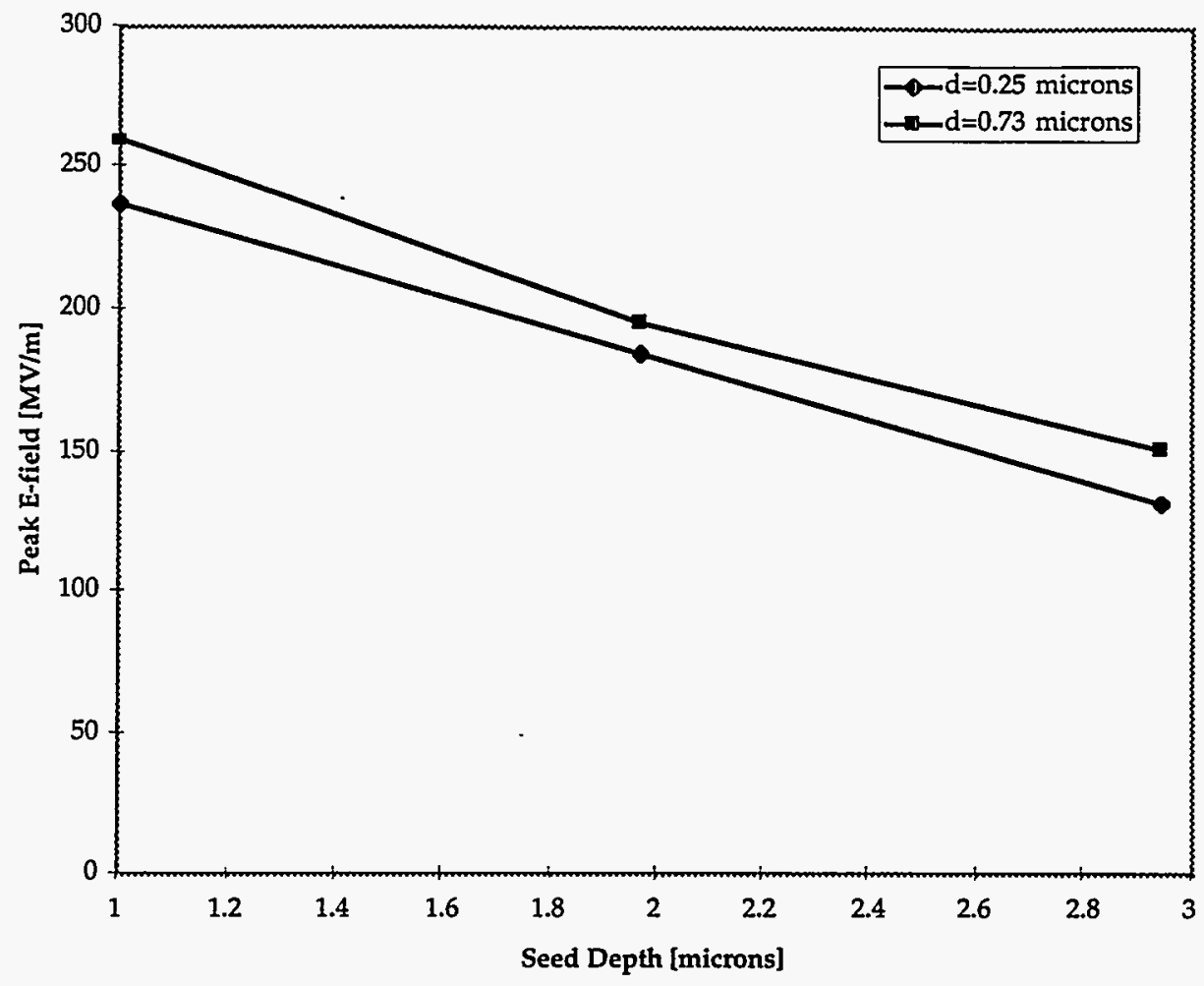

Figure 16. The peak steady-state electric field (in $\mathrm{MV} / \mathrm{m}$ ) as a function of $\mathrm{HfO}_{2}$ seed depth (in $\mu \mathrm{m}$ ) for two seed sizes (seed diameters $d=0.25 \mu \mathrm{m}$ and $d=0.73 \mu \mathrm{m}$ ).

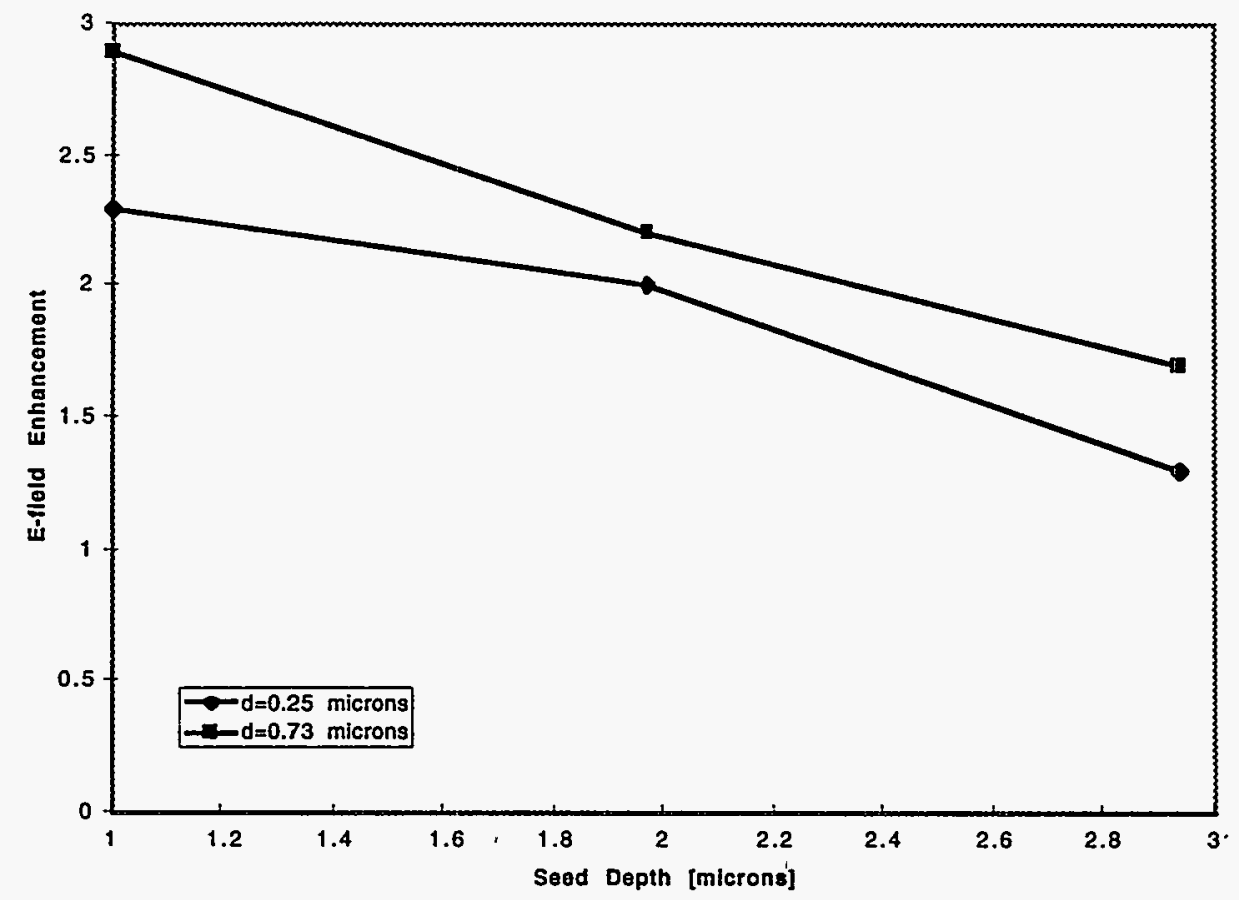

Figure 17. The electric field enhancement as a function of $\mathrm{HfO}_{2}$ seed depth (in $\mu \mathrm{m}$ ) for two seed sizes (seed diameters $d=0.25 \mu \mathrm{m}$ and $d=0.73 \mu \mathrm{m}$ ). 


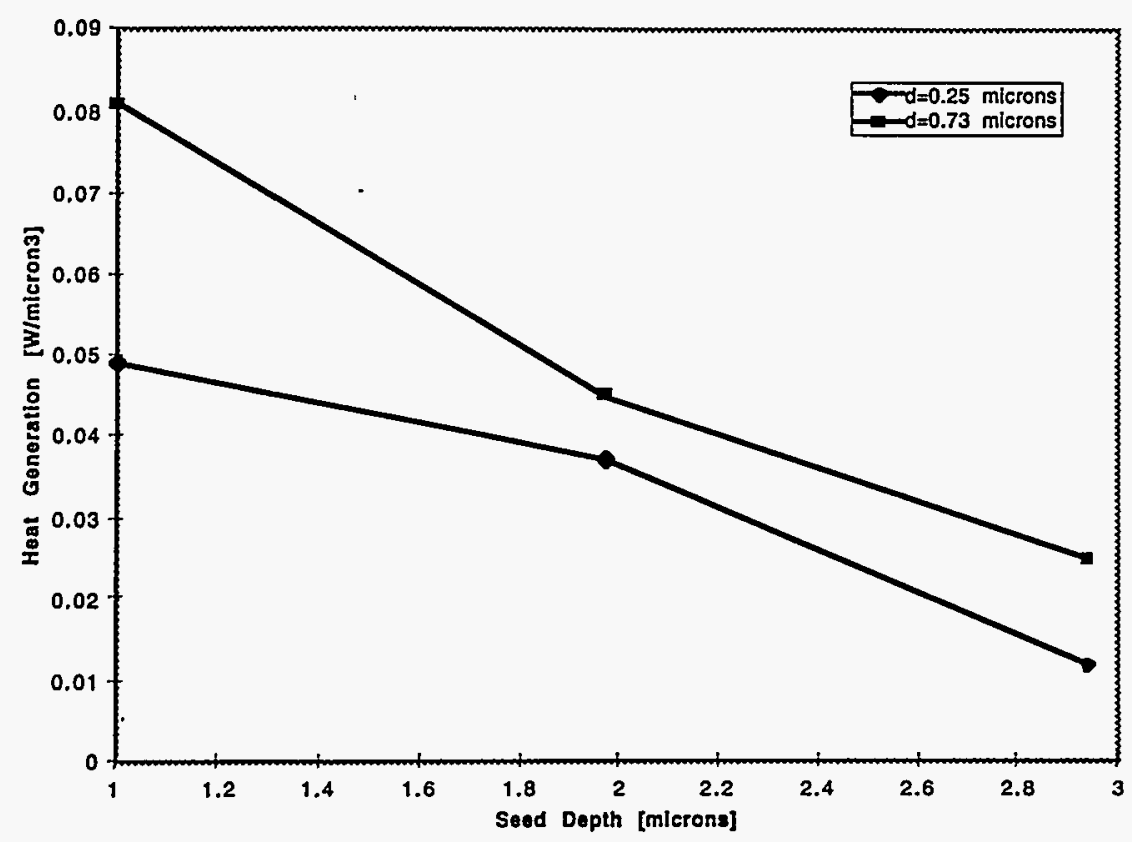

Normalized E-field

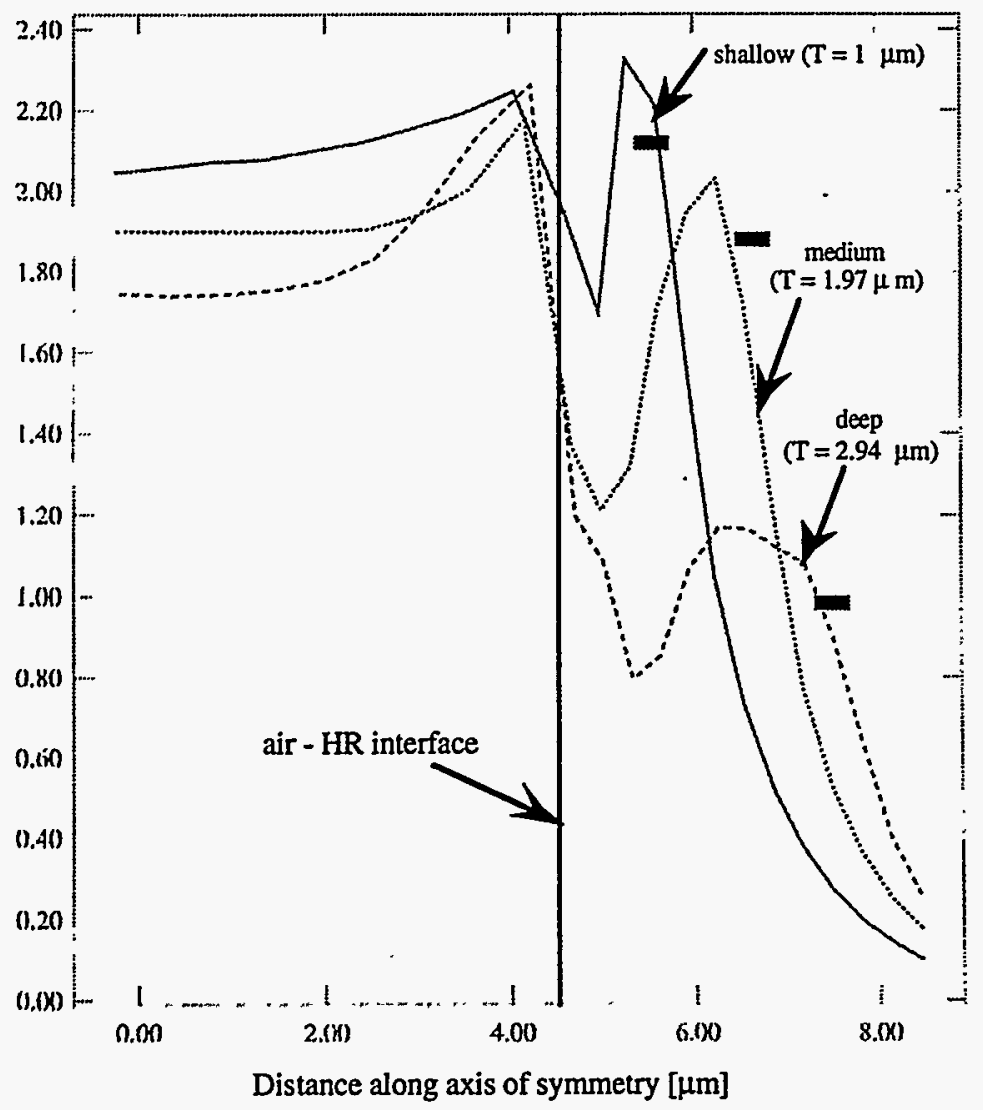

Figure 18. The peak heat generation (in $W / \mu \mathrm{m}^{3}$ ) as a function of $\mathrm{HfO}_{2}$ seed depth (in $\mu \mathrm{m}$ ) for two seed sizes (seed diameters $\mathrm{d}=0.25 \mu \mathrm{m}$ and $d=0.73 \mu \mathrm{m}$ ).
Figure 19. The envelopes of the normalized EFSW pattern (normalized to the input electric field) along the axis of symmetry $(\mathrm{r}=0 \mu \mathrm{m})$ at three different $\mathrm{HfO}_{2}$ seed depths for the small $(\mathrm{d}=0.25$ $\mu \mathrm{m})$ seed. The air-HR interface is indicated as is the location of the seed (the solid gray line segment) for each curve. 


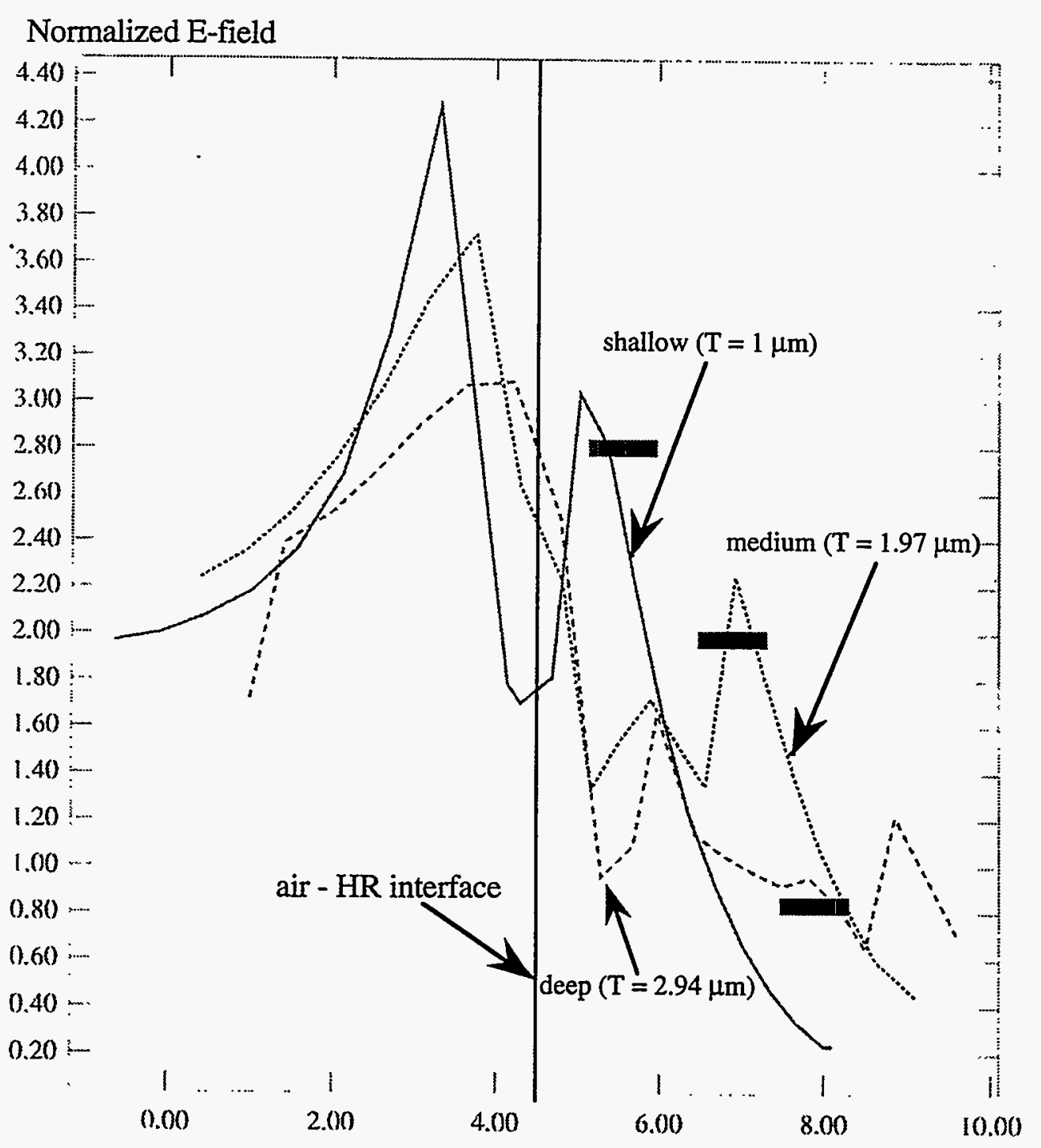

Distance along axis of symmetry $[\mu \mathrm{m}]$

Figure 20. The envelopes of the normalized EFSW pattern (normalized to the input electric field) along the axis of symmetry $(\mathrm{r}=0 \mu \mathrm{m})$ at three different $\mathrm{HfO}_{2}$ seed depths for the medium $(\mathrm{d}=0.73 \mu \mathrm{m})$ seed. The air-HR interface is indicated as is the location of the seed (the solid gray line segment) for each curve.

electric field decreases as the seed depth is increased. In addition, the location of the peaking in the electric field profile out in the air region moves closer to the air-HR interface as the seed depth is increased, and also gets lower in value.

The dependence of electric field distribution, electric field enhancement, and heat generation on seed diameter is also quite complex, and is, in general, not monotonic. This dependence is particularly complex for shallow seeds situated near the air/HR boundary. This may be due, in part, to the fact that for shallow seed depths, all but the 
smallest seed sizes create a significant nodule dome above the seed, exhibiting large curvature, which could lead to more pronounced microlens and resonance effects. However, for deep seeds ( $\mathrm{T} \geq 2.94 \mu \mathrm{m}$ ), the dependence of electric field, electric-field enhancement, and heat generation does appear to follow a monotonic trend, increasing with increasing seed diameter, as is shown in Figs. 21, 22, and 23, respectively. This indicates that for deeply embedded seeds, the seed diameter is the important factor affecting the electric-field enhancement.

Fig. 24 shows the envelopes of the normalized EFSW pattern (normalized to the input electric field) along the axis of symmetry $(\mathrm{r}=0 \mu \mathrm{m})$ for a deep seed $(\mathrm{T}=2.94 \mu \mathrm{m})$ for various seed diameters. The air-HR interface is indicated in the figure as is the location of the seed (the solid gray line segment) for each curve. It is apparent from Fig. 24 that the "hot spots" in the electric field pattern are outside the $\mathrm{HfO}_{2}$ seed and near the air-HR interface regardless of seed diameter, with the peak value increasing as the seed diameter increases. The peaking of the electric field profile out in the air region remains in essentially the same location as seed size is increased.

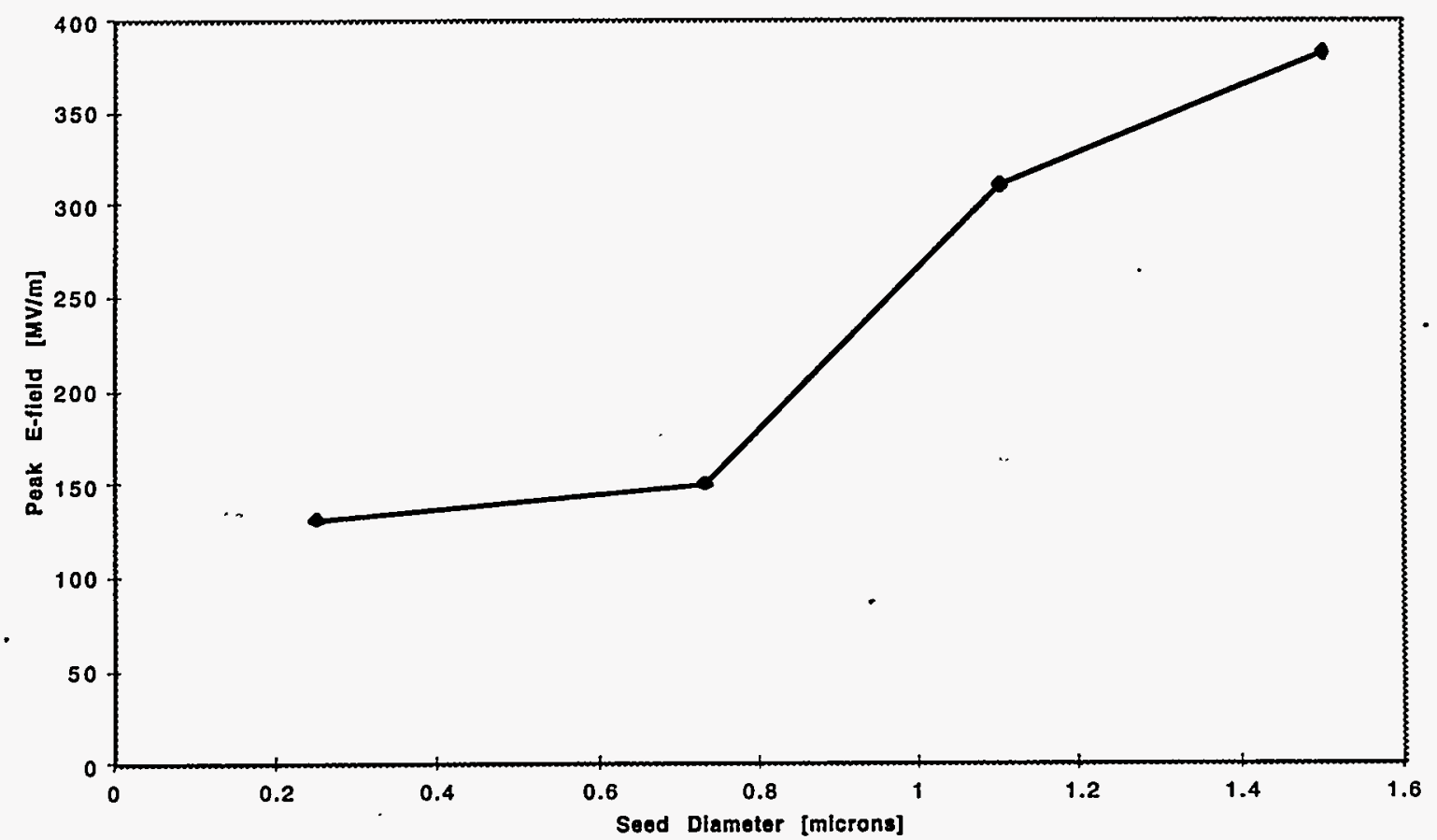

Figure 21. The peak steady-state electric field (in $\mathrm{MV} / \mathrm{m}$ ) as a function of $\mathrm{HfO}_{2}$ seed diameter (in $\mu \mathrm{m}$ ) for a depth, $\mathrm{T}$, of $2.94 \mu \mathrm{m}$. 


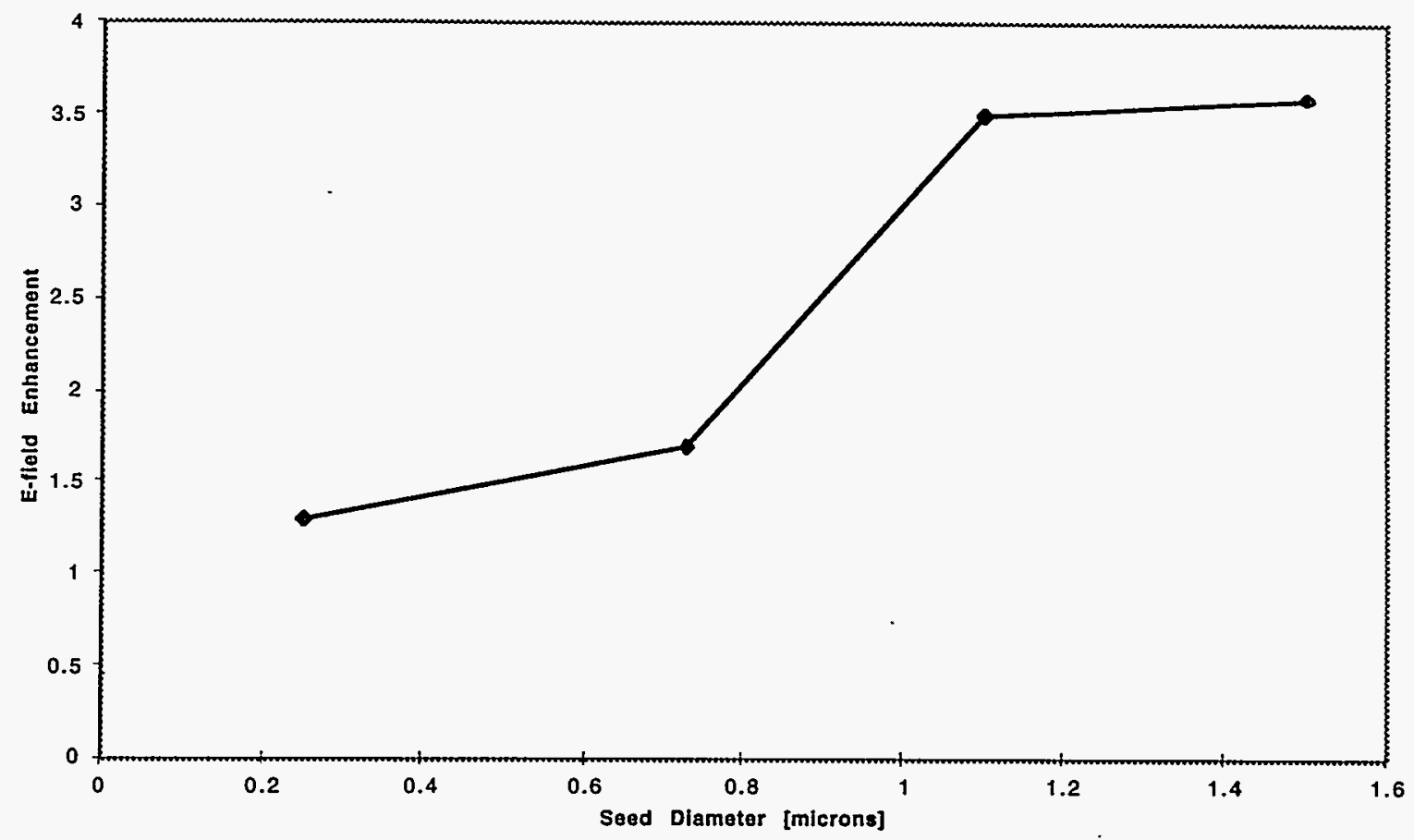

Figure 22. The electric field enhancement as a function of $\mathrm{HfO}_{2}$ seed diameter (in $\mu \mathrm{m}$ ) for a depth, $\mathrm{T}$, of $2.94 \mu \mathrm{m}$.

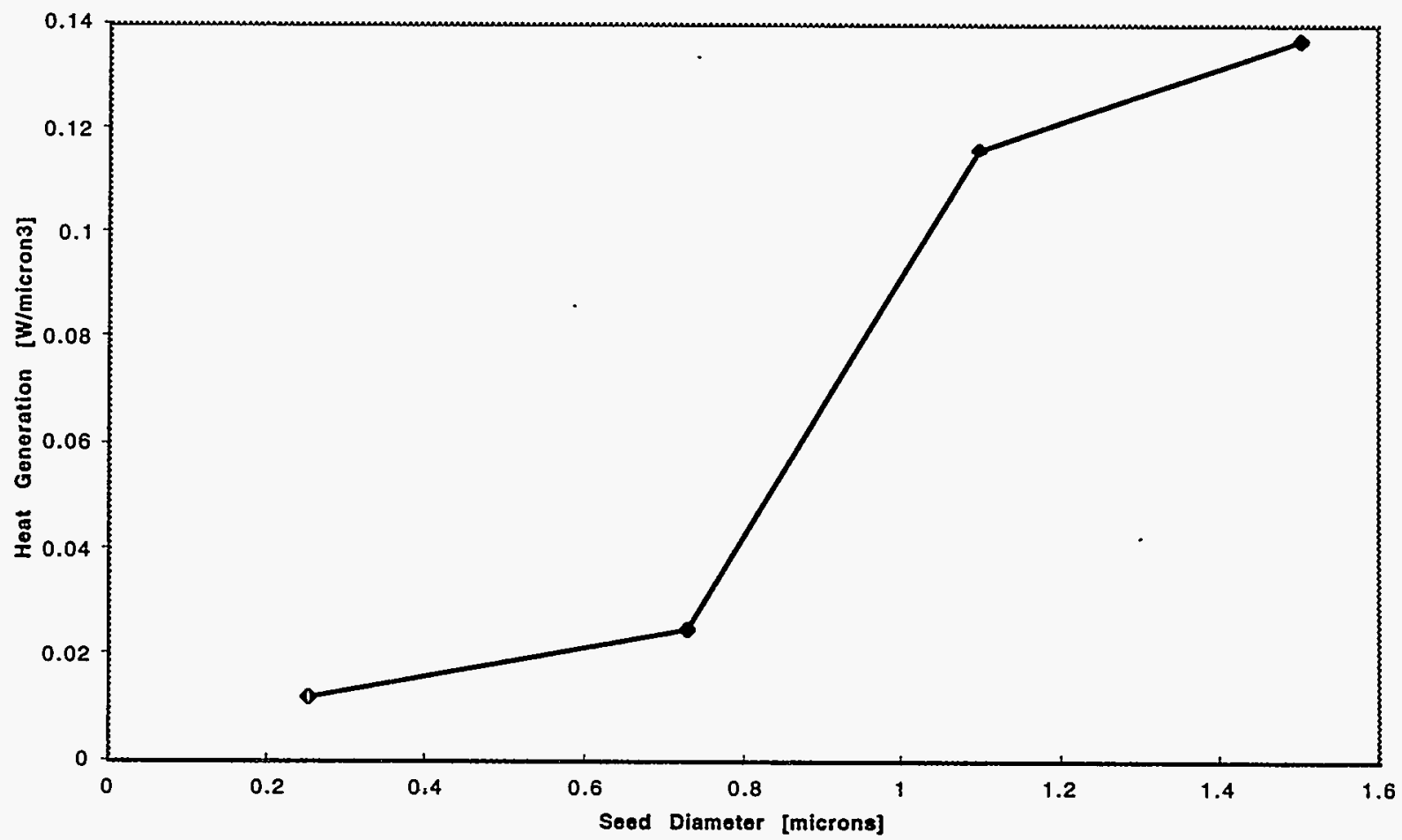

Figure 23. The peak heat generation (in $\mathrm{W} / \mu \mathrm{m}^{3}$ ) as a function of $\mathrm{HfO}_{2}$ seed diameter (in $\mu \mathrm{m}$ ) for a depth, $\mathrm{T}$, of $2.94 \mu \mathrm{m}$. 


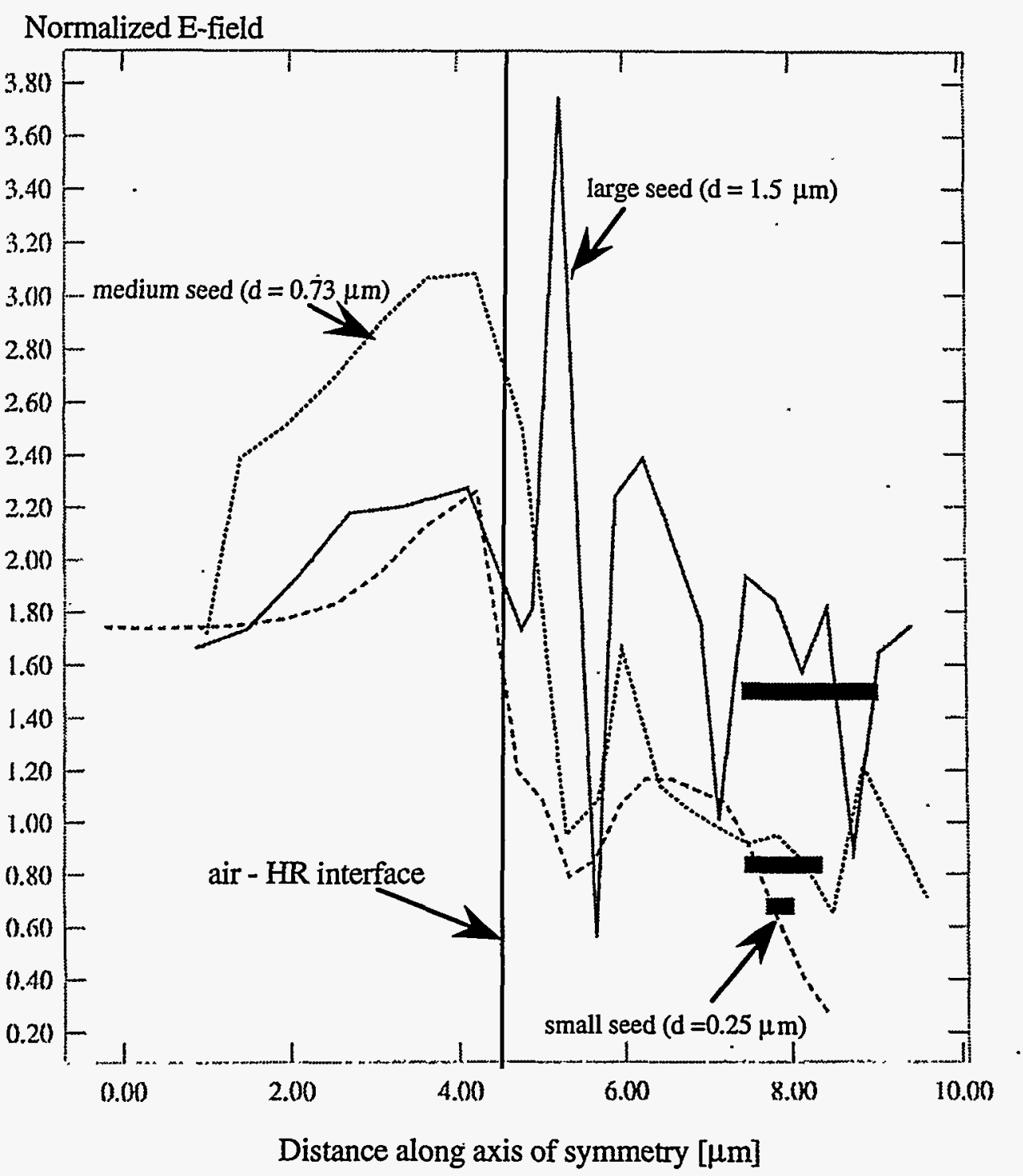

Figure 24. The envelopes of the normalized EFSW pattern (normalized to the input electric field) along the axis of symmetry $(\mathrm{r}=0 \mu \mathrm{m})$ for a deep seed $(\mathrm{T}=2.94 \mu \mathrm{m})$ for various $\mathrm{HfO}_{2}$ seed diameters. The airHR interface is indicated as is the location of the seed (the solid gray line segment) for each curve.

The dependence of electric field profile on seed material composition is illustrated in Fig. 25 which shows the envelopes of the normalized EFSW pattern (normalized to the input electric field) along the axis of symmetry $(r=0 \mu \mathrm{m})$ for a medium seed $(\mathrm{d}=0.73 \mu \mathrm{m})$ at medium depth $(\mathrm{T}=1.97 \mu \mathrm{m})$ for two seed material compositions: a $\mathrm{HfO}_{2}$ seed and a $\mathrm{SiO}_{2}$ seed. The electric field profiles are essentially identical for the two cases in regions away from the seed location which is to be expected since the geometries are identical except for seed composition. However, the electric field profiles are different in and near the seed location with the peak electric field being higher in the geometry containing the $\mathrm{SiO}_{2}$ seed. This may be due, in part, to 
resonance effects since the seed diameter is equal to one wavelength in $\mathrm{SiO}_{2}$. Although the dependence of electric field enhancement on defect geometry is, in general, quite complex, a good rule-of-thumb to follow is that geometries containing large, shallow seeds lead to the largest electric-field enhancements (or, equivalently, to the highest heat generation) while geometries containing small, deep seeds lead to the smallest electric field enhancements (or, equivalently, to the lowest heat generation).

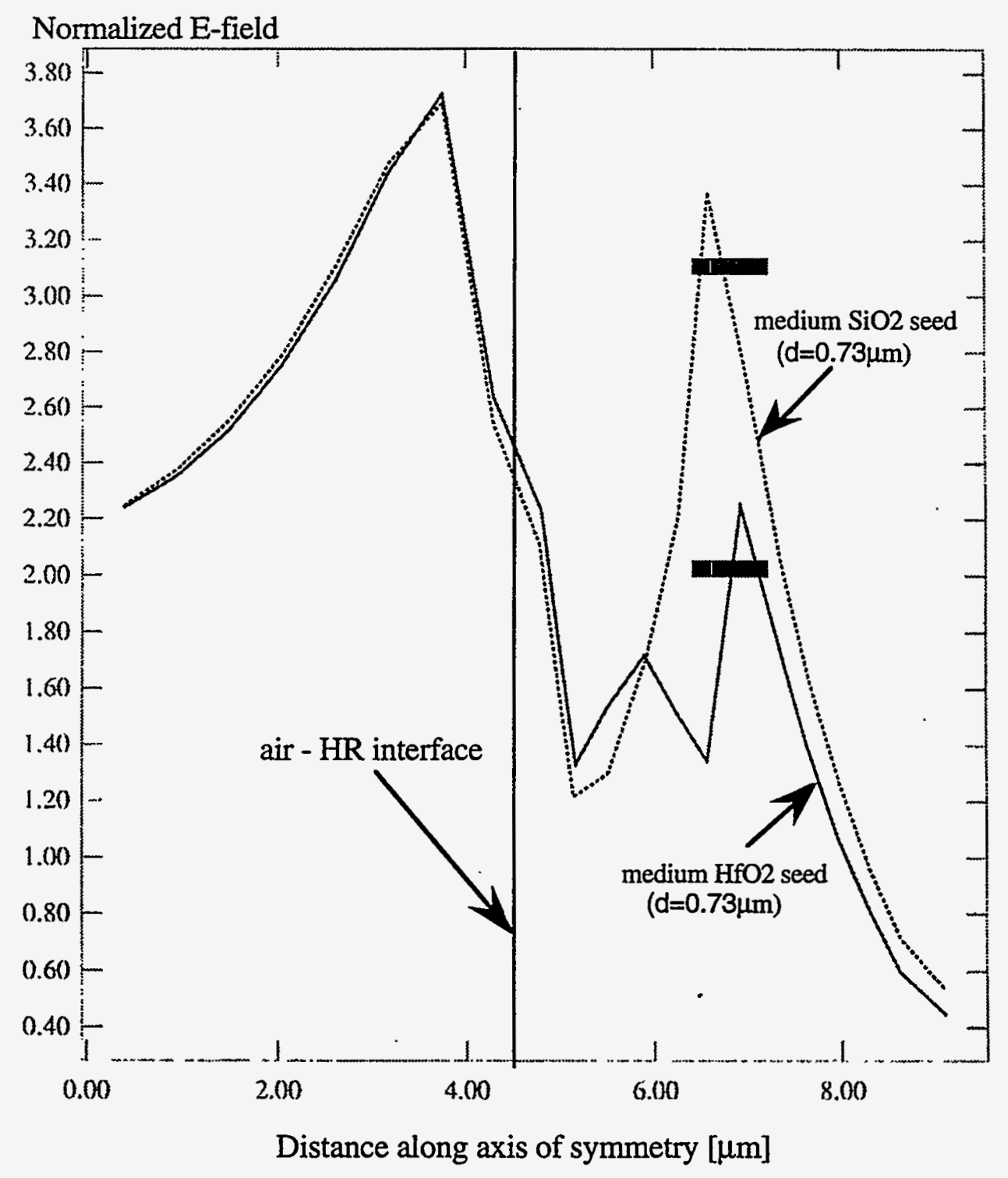

Figure 25. The envelopes of the normalized EFSW pattern (normalized to the input electric field) along the axis of symmetry $(r=0 \mu \mathrm{m})$ for a medium seed $(\mathrm{d}=0.73 \mu \mathrm{m})$ at medium depth $(\mathrm{T}=1.97 \mu \mathrm{m})$, for two seed material compositions: a $\mathrm{HfO}_{2}$ seed and a $\mathrm{SiO}_{2}$ seed. The air- $\mathrm{HR}$ interface is indicated in the figure as is the location of the seed (the solid gray line segment) for each curve. 


\section{Conclusions}

The results of a 2-1/2-D EM modeling effort, investigating the dependence of the electric field distribution, electric field enhancement, and heat generation on rotationally-symmetric nodular defect geometries in $\mathrm{HR}_{\mathrm{HfO}} / \mathrm{SiO}_{2}$ dielectric stack mirrors are summarized in this report. The 2-1/2-D FDTD EM code, AMOS, was used to perform the EM modeling, and a normal-incidence, linearly-polarized, sinusoidallyvarying (at $2.8302 \times 10^{14} \mathrm{~Hz}$, corresponding to an optical wavelength of $1.06 \mu \mathrm{m}$ ) plane wave was assumed as the input source. We developed a new output diagnostic for the AMOS solver, enabling us to obtain the single-frequency Fourier Transform coefficients of the electric field components at every node throughout a 2-D planar r-z region. By relating the single-frequency Fourier Transform coefficients of the electric field components to the peak steady-state amplitudes of the electric field components, we were able to calculate the peak steady-state electric field everywhere within the defect geometry. In addition, we developed the capability of coupling the 2-1/2-D EM modeling results into 2-D thermal-mechanical codes such as the commercially available COSMOS/M code or LLNL's 2-D NIKE2D/TOPAZ2D code suite, as well as into LLNL's 3-D thermal-mechanical code suite, NITO3D (comprised of the loosely-coupled NIKE3D and TOPAZ3D codes).

A matrix of 23 different nodular defect geometries was modeled, investigating the effects of seed size, depth and material composition on electric-field enhancement. The seed diameters ranged from $0.1 \mu \mathrm{m}$ to $1.5 \mu \mathrm{m}$, while the seed depth ranged from $0.69 \mu \mathrm{m}$ to $2.94 \mu \mathrm{m}$. All geometries assumed a $\mathrm{HfO}_{2}$ seed except for one, geometry \#6, which assumed a $\mathrm{SiO}_{2}$ seed. It was determined that the highest electric field enhancements occurred along the axis of symmetry, with the highest electric field enhancement of 6.4 reported for the largest, most shallow $\mathrm{HfO}_{2}$ seed $(\mathrm{d}=1.5 \mu \mathrm{m}$ and $\mathrm{T}$ $=1 \mu \mathrm{m}$ ), and the lowest electric field enhancement of 1.3 reported for the smallest, most deeply embedded $\mathrm{HfO}_{2}$ seed $(\mathrm{d}=0.25 \mu \mathrm{m}$ and $\mathrm{T}=2.94 \mu \mathrm{m})$. Note that an electric field enhancement of 6.4 would lead to an enhancement in the heat generation $\left(\sigma \mathrm{E}^{2}\right)$ of a factor of 41. It was determined that for small seeds $(\mathrm{d} \leq 1 \mu \mathrm{m})$, the important factor influencing electric field enhancement was the seed depth, with an increasing seed depth leading to a decreasing electric field enhancement. On the other hand, for deeply embedded seeds $(T \geq 2.94 \mu \mathrm{m}$ ), the important factor influencing the electric field enhancement was the seed size, with an increasing seed diameter leading to an increasing electric field enhancement. Trends for some of the other nodular defect geometries were not as clear, indicating that perhaps other complex effects such as 
resonance or other higher-order EM interactions may be the dominant influences on electric-field enhancements for those cases. These results predict that, as a general ruleof-thumb, geometries containing large, shallow seeds lead to the largest electric-field enhancements (or, equivalently, to the highest heat generation) while geometries containing small, deep seeds lead to the smallest electric field enhancements (or, equivalently, to the lowest heat generation). These conclusions are in close agreement with the EM modeling results reported by DeFord ${ }^{3}$. In addition, it was determined experimentally by Kozlowski ${ }^{4}$ that nodular defects with large dome heights (particularly those above $0.6 \mu \mathrm{m}$ ) were found to be most susceptible to laser damage, which is supported by our conclusion that large, shallow defects lead to the highest electric field enhancement and, therefore, the highest heat generation.

\section{Future Work}

Future work will extend the electro-mechanical modeling effort to the full 3-D regime, allowing for both generalized sources (arbitrary angle-of-incidence and arbitrary polarization) and generalized defect geometries (asymmetric geometries). We will use the 3-D frequency-domain EM code, EIGER (currently under development at LLNL by Sharpe, et al.), to compute the steady-state electric field distribution throughout these 3-D defect geometries. We will continue to develop our EM-thermalmechanical coupled code capability to couple the spatial distribution of the steady-state electric field from EIGER to the 3-D thermal-mechanical code suite, NITO3D. Solution convergence studies will be performed as part of the code-coupling effort. The 3-D thermal-mechanical code suite, NITO3D will then be used to analyze heat diffusion as well as the spatial and temporal distributions of temperature and stresses in order to achieve a fully 3-D electro-mechanical model of nodule failure for various defect geometries.

The EIGER code has already been benchmarked against analytical solutions such as the perfect mirror stack for normal incidence as well as for a $45^{\circ}$ angle-of-incidence. Prior to proceeding with modeling a new matrix of asymmetric, oblique-incidence nodular defect geometries, the 3-D EM and thermal-mechanical codes will be benchmarked using the previously analyzed rotationally-symmetric, defect geometries (using normal-incidence illumination). Because the EIGER code uses stratified media Green's functions to obtain exact solutions for the fields out in the planar region of the dielectric stack mirror, only the parabolic nodular defect region of the geometry needs to be defined using surface meshes. The entire parabolic surface of the nodular defect is 
meshed as is the defect dome surface at the air-HR interface, and the surfaces between the adjacent layers of $\mathrm{HfO}_{2}$ and $\mathrm{SiO}_{2}$ within the defect region itself. The EIGER code will enable us to calculate more general 3-D nodular defect geometries allowing us to study a larger subset of experimentally-observed classes of defects.

\section{Acknowledgments}

The authors would like to acknowledge T. A. Reitter and A. B. Shapiro for their work in the TOPAZ/NIKE thermal-mechanical modeling effort for the 2-1/2-D nodular defect study, F. Y. Genin for his experimental investigation and insight into material properties and coating failure mechanisms, and C. J. Stolz for providing the micrograph data shown in Figure 7. We would also like to acknowledge useful discusșions with J. F. DeFord of the MacNeil-Schwendler Company, Milwaukee, Wisconsin. In addition, we wish to acknowledge Teresa Swatloski of TGV, Inc., Santa Cruz, California for her contributions in computer science support for this project, as well as P. P. Weidhaas of LLNL for his contributions in developing the PREAMOS preprocessor. We would also like to acknowledge John Creighton, a summer employee at LLNL, for his contributions in generating the 3-D surface meshes for the rotationally-symmetric defect geometries. We would like to acknowledge R. M. Sharpe and J. B. Grant of LLNL for useful discussions about the EIGER code. Finally, our thanks to Lyn Ahboltin for her excellent administrative support. 